\title{
ATP Binding to Synaspsin IIa Regulates Usage and Clustering of Vesicles in Terminals of Hippocampal Neurons
}

\author{
Yoav Shulman, ${ }^{1,2 \star}$ Alexandra Stavsky, ${ }^{1,2 *}$ Tatiana Fedorova, ${ }^{3}$ Dan Mikulincer, ${ }^{1}$ Merav Atias, ${ }^{1}$ Igal Radinsky, ${ }^{1}$ \\ Joy Kahn, ${ }^{1}$ Inna Slutsky, ${ }^{3}$ and ${ }^{-D a n i e l ~ G i t l e r ~}{ }^{1,2}$ \\ ${ }^{1}$ Department of Physiology and Cell Biology, Faculty of Health Sciences, and 'Zlotowski Center for Neuroscience, Ben-Gurion University of the Negev, Beer- \\ Sheva 84105, Israel, and 3 ${ }^{3}$ epeartment of Physiology and Pharmacology, Sackler Faculty of Medicine, and Sagol School of Neuroscience, Tel Aviv University, \\ 69978 Tel Aviv, Israel
}

Synaptic transmission is expensive in terms of its energy demands and was recently shown to decrease the ATP concentration within presynaptic terminals transiently, an observation that we confirm. We hypothesized that, in addition to being an energy source, ATP may modulate the synapsins directly. Synapsins are abundant neuronal proteins that associate with the surface of synaptic vesicles and possess a well defined ATP-binding site of undetermined function. To examine our hypothesis, we produced a mutation (K270Q) in synapsin IIa that prevents ATP binding and reintroduced the mutant into cultured mouse hippocampal neurons devoid of all synapsins. Remarkably, staining for synaptic vesicle markers was enhanced in these neurons compared with neurons expressing wild-type synapsin IIa, suggesting overly efficient clustering of vesicles. In contrast, the mutation completely disrupted the capability of synapsin IIa to slow synaptic depression during sustained $10 \mathrm{~Hz}$ stimulation, indicating that it interfered with synapsin-dependent vesicle recruitment. Finally, we found that the K270Q mutation attenuated the phosphorylation of synapsin IIa on a distant PKA/CaMKI consensus site known to be essential for vesicle recruitment. We conclude that ATP binding to synapsin IIa plays a key role in modulating its function and in defining its contribution to hippocampal short-term synaptic plasticity.

Key words: ATP; phosphorylation; short-term plasticity; synapsin; synaptic vesicle; vesicle pools

\section{Introduction}

Synaptic transmission is the basis of interneuronal communication. The strength of synaptic connections is modulated at various timescales in a process termed synaptic plasticity (Zucker and Regehr, 2002; Regehr, 2012). Because plasticity plays a significant role in neuronal computation (Abbott and Regehr, 2004), it remains a central topic of investigation. Presynaptic mechanisms, including those involving vesicle dynamics, contribute substantially to short-term plasticity. In particular, recycling of vesicles, their subdivision into pools, and the capability of presynaptic terminals to use them have attracted significant interest (Südhof, 2004; Denker and Rizzoli, 2010; Neher, 2010; Rizzoli, 2014).

The synapsins are a three-gene family of neuron-specific proteins that interface between the surface of synaptic vesicles and

\footnotetext{
Received March 9, 2014; revised 0ct. 24, 2014; accepted Nov. 18, 2014.

Author contributions: Y.S., A.S., I.S., and D.G. designed research; Y.S., A.S., T.F., D.M., M.A., I.R., and J.K. performed research; Y.S., A.S., T.F., D.M., M.A., J.K., I.S., and D.G. analyzed data; D.G. wrote the paper.

This work was supported by the Israel Science Foundation (Grant 1427/12 to D.G.) and the European Research Council (Starting Grant 281403 to I.S.). We thank Hiromi Imamura for the kind gift from ATeam1.03 and ATeam1.03 ${ }^{\text {R122K/R126K }}$, Atsushi Miyawaki for Venus, Thomas Kuner for AAV vectors encoding EGFP-Synapsin lla and Synaptophysin I-EGFP, Jürgen Kleinschmidt for kindly sharing the AAV system, Fabio Benfenati for the C57BL6backcrossed synapsin TKO mice, and llya Fleidervish for excellent expert advice and opinions.

The authors declare no competing financial interests.

*Y.S. and A.S. contributed equally to this work.

Correspondence should be addressed to Dr. Daniel Gitler, Department of Physiology and Cell Biology, Faculty of Health Sciences, Ben-Gurion University of the Negev, Beer-Sheva 84105, Israel. E-mail: gitler@bgu.ac.il.

DOI:10.1523/JNEUROSCI.0944-14.2015

Copyright $\odot 2015$ the authors $\quad 0270-6474 / 15 / 350985-14 \$ 15.00 / 0$
}

the cytoskeleton (Cesca et al., 2010). The association of synapsins with these components is modulated by phosphorylation (Jovanovic et al., 2001; Chi et al., 2003; Verstegen et al., 2014) and, because neuronal activity defines the balance between kinase/ phosphatase pairs (Kim and Ryan, 2013), synapsins associate between neuronal activity and synaptic plasticity (Cesca et al., 2010). For example, both synapsins I and II affect synaptic depression and its recovery (Samigullin et al., 2004; Menegon et al., 2006; Baldelli et al., 2007; Gitler et al., 2008; Verstegen et al., 2014), whereas synapsin I participates in paired-pulse modulation (Rosahl et al., 1995).

More than a decade ago, it was reported that the synapsins bind ATP (Esser et al., 1998; Hosaka and Südhof, 1998b). Moreover, the residues participating in ATP binding were identified (Esser et al., 1998). Nonetheless, the implications of this finding were not followed up. We hypothesize that activity-dependent spatiotemporal alterations in the synaptic ATP concentration $\left([\mathrm{ATP}]_{\text {syn }}\right)$ modulate synapsin function. Specifically, we propose that $[\mathrm{ATP}]_{\text {syn }}$ affects the balance between vesicle clustering and mobilization in a synapsin-dependent manner. Notably, ATP allosterically modulates various proteins in diverse physiological settings, such as the $\mathrm{K}_{\mathrm{ATP}}$ potassium channels involved in insulin secretion by pancreatic $\beta$ cells (Henquin, 2000), ClC-2 anion channels (Stölting et al., 2013), and the enzyme serine racemase (Marchetti et al., 2013).

Brain function, specifically synaptic activity, is a major consumer of energy (Rangaraju et al., 2014). Most synapses contain 
mitochondria, strategically placed to supply these energy needs (MacAskill et al., 2010; Harris et al., 2012). However, it is not a priori clear that alterations in energy expenditure translate into local changes in $[\mathrm{ATP}]_{\mathrm{syn}}$. For example, ATP levels remain steady during intense cardiac pacing (Balaban et al., 1986) due to calcium-dependent upregulation of ATP production (Chouhan et al., 2012; Glancy and Balaban, 2012). Therefore, we verified that $[\mathrm{ATP}]_{\text {syn }}$ changes under activity regimes relevant to synapsin function, as reported recently (Rangaraju et al., 2014). We examined the consequences of ATP binding by synapsins by reintroducing synapsin IIa mutated at an amino acid essential for ATP binding into synapsin-null neurons. Specifically, we probed vesicle clustering at rest, vesicle utilization during intense synaptic activity, and effects on synapsin phosphorylation. Our results indicate that ATP binding by synapsin IIa is profoundly significant for synapsin-dependent synaptic plasticity and for defining the resting structure of the presynaptic terminal.

\section{Materials and Methods}

Solutions, materials and antibodies

Solutions. Extracellular saline contained the following (in mM): $150 \mathrm{NaCl}$, $3 \mathrm{KCl}, 20$ glucose, $10 \mathrm{HEPES}, 2 \mathrm{CaCl}_{2}, 2 \mathrm{MgCl}_{2}, \mathrm{pH}$ adjusted to 7.35 with $\mathrm{NaOH}, 310$ mOsm. Intracellular solution contained the following (in mM): 113 potassium gluconate, $15 \mathrm{NaCl}, 6 \mathrm{MgCl}_{2}, 20 \mathrm{HEPES}, 2$ EGTA, 5 $\mathrm{Na}_{2} \mathrm{ATP}, 0.3 \mathrm{NaGTP}$, pH adjusted to 7.3 with $\mathrm{KOH}, 295$ mOsm. Hyperkalemic saline was the same as extracellular saline, but $87 \mathrm{~mm} \mathrm{NaCl}$ was replaced with $\mathrm{KCl}$. ATP-depleting saline was the same as extracellular saline, $20 \mathrm{~mm}$ glucose was replaced with 2-deoxy-D-glucose and $1 \mu \mathrm{m}$ oligomycin was added. Tyrode's solution contained the following (in mM): $145 \mathrm{NaCl}, 3 \mathrm{KCl}, 15$ glucose, 10 HEPES, $1.2 \mathrm{CaCl}_{2}, 1.2 \mathrm{MgCl}_{2}, \mathrm{pH}$ adjusted to 7.4 with $\mathrm{NaOH}$. Lysis buffer contained $\mathrm{Ca}^{2+} / \mathrm{Mg}^{2+} \mathrm{PBS}$ (Biological Industries) supplemented with 0.1\% Triton X-100a and 1:50 Boehringer Complete Protease Inhibitor Mixture (Roche Molecular Biochemicals).

Materials. 6,7-Dinitroquinoxaline-2,3(1H,4H)-dione (DNQX), picrotoxin, 2-deoxy-D-glucose, oligomycin were from Sigma-Aldrich. Bafilomycin A, N-[2-[[3-(4-Bromophenyl)-2-propenyl]amino]ethyl]5-isoquinoline-sulfonamide (H89) was from Enzo Life Sciences. Restriction enzymes and T4-ligase were from Fermentas/Thermo Scientific Life Science Research.

Antibodies. Rabbit polyclonal anti-Synaptobrevin 2 (1:600), Rabbit polyclonal anti-Synapsin II (IB: 1:2500), Guinea pig polyclonal antiSynaptophysin I (1:1000) were from Synaptic Systems. Rabbit polyclonal anti phospho-S9 synapsin I (1:200) was from Phosphosolutions. The mouse monoclonal GAD-6 antibody against Glutamic acid decarboxylase of $65 \mathrm{KDa}$ (GAD-6, 1:1000), developed by D.I. Gottlieb, was obtained from the Developmental Studies Hybridoma Bank (DSHB) developed under the auspices of the National Institute of Child Health and Human Development and maintained by The University of Iowa Department of Biology. Donkey anti-mouse IgG and donkey anti-rabbit IgG, labeled with Northern Lights 637 or 557, respectively (1:1000) were from R\&D Systems. Affinity-purified goat anti-rabbit IgG, labeled with Cy3 (1:100), and goat anti-guinea pig labeled with DyLight 649 (1:1000) were from Jackson ImmunoResearch Laboratories. Goat anti-rabbit IgG coupled to horseradish peroxidase (IB: 1:5000) was from Pierce/Thermo Scientific.

\section{Mice and neuronal cultures}

Synapsin triple knock-out (TKO) mice (Gitler et al., 2004a), backcrossed onto the C57BL6 background (Boido et al., 2010), were grown at the Ben-Gurion University mouse facility. C57BL6 wild-type (WT) controls were obtained from Harlan Laboratories. Animals were treated in accordance with the guidelines of the Ben-Gurion University Institutional Committee for Ethical Care and Use of Animals in Research. Dense or autaptic hippocampal cultures from postnatal day 0 (P0)-P2 pups of either sex were performed essentially as described previously (Gitler et al., 2004b). Cells were grown for 12-17 d before imaging and for $10-14 \mathrm{~d}$ for electrophysiological experiments. Experiments were performed at room temperature.

\section{Molecular constructs, transfections, and infections}

Viral particles were prepared as described previously (Groh et al., 2008) in HEK cells using both the pD1 and pD2 helper plasmids. cDNAs of interest (EGFP/TagBFP-Synapsin IIa, Synaptophysin I-EGFP/TagBFP, Synaptophysin I-ATeam1.03, Synaptophysin I-ATeam 1.03 R122K/R126K) were subcloned by restriction/ligation into a plasmid containing adenoassociated virus 2 (AAV2) inverted terminal repeats flanking a cassette consisting of the $1.1 \mathrm{~kb}$ cytomegalovirus enhancer/chicken $\beta$-actin promoter, the woodchuck posttranscriptional regulatory element, and the bovine growth hormone polyA. PCR-assisted site-directed mutagenesis to produce the $\mathrm{K} 270 \mathrm{Q}$ mutation was performed using the mutagenesis primers (sense: 5'-GTTTCCTGTCGTGGTGCAGATTGGCCATGCTC AC-3', antisense: 5'-GTGAGCATGGCCAATCTGCACCACGACAGG AAAC- $3^{\prime}$ ), followed by DpnI digestion. The S10A mutation was performed similarly (sense: $5^{\prime}$-CTGAGGCGCCGGCTGGCGGACAGCAGCTTCA TTG-3' ${ }^{\prime}$, antisense: 5' $^{\prime}$-CAATGAAGCTGCTGTCCGCCAGCCGGCGCC TCAG-' $\left.^{\prime} 3\right)$. Final products were verified by sequencing. Virions were added to neurons at $5 \mathrm{~d}$ in vitro and cells were incubated for at least $4 \mathrm{~d}$ before experimentation. Virion titer was individually adjusted to produce $50-90 \%$ infection efficiency. EGPF was from Clontech; Venus was a kind gift from Atsushi Miyakawi (Nagai et al., 2002); ATeam1.03 and ATeam 1.03 ${ }^{\text {R122k/R126K }}$ were a kind gift from Hiromi Imamura (Imamura et al., 2009); and TagBFP was from Evrogen.

\section{Western blot analysis}

HEK-T cells were transfected with EGPF-SynIIa or EGFP-SynIIa-K270Q expression plasmids using the calcium-phosphate precipitation method. After $24 \mathrm{~h}$, cells were trypsinized, washed twice in PBS, resuspended in $300 \mu \mathrm{l}$ of lysis buffer, and homogenized. The homogenate was cleared by centrifugation at $1500 \times \mathrm{g}$ for $5 \mathrm{~min}$ at $4^{\circ} \mathrm{C}$ and the supernatant was frozen and stored at $-80^{\circ} \mathrm{C}$ for future use. Protein concentrations were determined using the Bradford assay (Bio-Rad). Equal protein quantities were subjected to SDS-PAGE gel electrophoresis and immunoblot analysis was performed using an anti-synapsin II antibody (1:2500) PBS/5\% low-fat milk, followed by incubation with a secondary anti-rabbit-IgG antibody coupled to HRP (1:5000). Detection was done with the EZ-ECL Chemiluminescence Detection kit for HRP (Biological Industries) on an ImageQuant LAS 4000 digital imaging system (General Electric Healthcare Bio-Sciences).

\section{Fluorescence microscopy}

Fluorescence recovery after photobleaching (FRAP) experiments were performed essentially as described previously (Orenbuch et al., 2012a), except the imaging intervals were $30 \times 15 \mathrm{~s}$, then $10 \times 90 \mathrm{~s}$, and finally $5 \times 150 \mathrm{~s}$ for a total of $35 \mathrm{~min}$. Imaging was done on a C1si spectral confocal microscope driven by the EZC1 software package (Nikon). The scanhead was mounted on a Nikon FN1 microscope, equipped with a 1.0 numerical aperture (NA) $60 \times$ water-dipping objective. The $488 \mathrm{~nm}$ line of a multiline $65 \mathrm{~mW}$ argon laser was used for excitation of EGFP, emissions were filtered with a $510-560 \mathrm{~nm}$ band-pass filter (Chroma). TagBFP was excited using a $405 \mathrm{~nm}$ solid-state diode laser and emissions were detected using the spectral detector of the C1si. Experiments were performed on at least three independent cultures. Other fluorescence measurements were performed on a Nikon TiE inverted microscope driven by the NIS-elements software package (Nikon). The microscope was equipped with a CoolSnap HQ2 14bit CCD camera (Roper Scientific), a $40 \times 0.75$ NA Super Fluor objective, a $60 \times 1.4$ NA oil-immersion apochromatic objective (Nikon), a perfect-focus mechanism (Nikon), and EGFP, EYFP, and Cy3 TE-series optical filter sets (Chroma) as well as BFP and Cy5 filter sets (Semrock).

\section{Semiquantitative synaptic immunofluorescence}

Neurons were fixed with $4 \%$ paraformaldehyde (EMS) in PBS for $10 \mathrm{~min}$, rinsed with PBS, permeabilized with $0.1 \%$ Triton X-100 in PBS for 2 min, blocked with 5\% powdered milk in PBS for $1 \mathrm{~h}$, rinsed, incubated with the primary antibody for $1 \mathrm{~h}$, rinsed, incubated with the secondary antibody for $1 \mathrm{~h}$, rinsed, and mounted in Immu-mount (Thermo Fisher Scientific). To allow for semiquantitative comparison of immunostaining intensity of synapses, experimental and control groups were processed under identical conditions using the same reagents. Synapses were 
detected semiautomatically using an in-house iterative algorithm based on serially decreasing to thresholds similar to those (Bergsman et al., 2006) implemented in NIS-elements. Fluorescence values for each synapse were obtained from an area of $2 \times 2$ pixels located on its center of mass and an image average was generated. Because intensity values of the controls can vary from session to session, a normalization value was determined from the control experiments of each session and this value was used to normalize all images acquired during that session. Puncta that were positive for GAD65 were deemed GABAergic; otherwise, they were considered glutamatergic. To perform analysis reliably on the synaptobrevin 2 (syb2) or synaptophysin I (SypI) content of synapsinexpressing puncta (see Fig. 4), an exclusion threshold was set at twice the background fluorescence in images of tagged synapsins.

\section{Calculation of the synaptic targeting factor and width}

The targeting factor of synapsin or synaptophysin I (Gitler et al., 2004a) was measured per synapse in neurons expressing EGFP-tagged synaptic proteins and soluble TagBFP. The fluorescence was measured in the synaptic puncta $(S y n)$ and in the axon. The ratio between synaptic and axonal signals in the green channel $(g)$ describes the enrichment of synapsin in the synapse, whereas the ratio in the blue channel $(b)$ corrects for volume differences in these two locales. Lack of targeting is expressed as 0 by subtracting 1 from the result. Values larger than 0 indicate that a protein is enriched in synapses.

$$
T F=\frac{\text { Syn }_{g} / \text { Axon }_{g}}{S y n_{b} / \text { Axon }_{b}}-1
$$

The fluorescence values were measured by drawing a line through each synapse, extracting the line profile and fitting it with a Gaussian function centered on the synapse:

$$
G(x)=y_{0}+A e^{-\frac{\left(x-x_{c}\right)^{2}}{2 w^{2}}}
$$

where $y_{0}$ is the fluorescence of the axon, $x_{\mathrm{c}}$ is the center of the punctum, $w$ is the SD (width) of the Gaussian, and $A$ is the fluorescence attributed to the vesicle cluster within the synapse (each corrected for the imaging background). Fitting was performed by the least-squares error method using Origin (OriginLab). The synaptic fluorescence was calculated as the mean signal within the line segment Syn $=X_{c} \pm w$, whereas the fluorescence of the axon was the mean signal of the line segment $A x<X_{c}$ $-3 * w$. Targeting indices were averaged per image and then across images. To quantify the width of the synaptic puncta, we calculated the full-width-at-half-maximum (FWHM) of the aforementioned Gaussian (Orenbuch et al., 2012b) as follows:

$$
F W H M=2 w \sqrt{\ln 4}
$$

Synaptic 'clustering index'

The clustering index of synapsin was assessed from neurons expressing EGFP-tagged synapsin, which where immunostained for syb2 or sypI and GAD65. Synapses stained for GAD65 were excluded. After correcting for background, the syb2/sypI fluorescence in each synapse was plotted as a function of the EGFP fluorescence and the resulting graph was fitted with a linear function. The slope of the function was defined as the per-image clustering index. Clustering indices per condition were compared across images.

\section{Fluorescence resonance energy transfer imaging and analysis}

Hippocampal neurons were imaged using a FV1000 spectral Olympus confocal microscope using a $60 \times 1.2 \mathrm{NA}$ water-immersion objective. Image acquisition parameters were optimized for maximal signal-tonoise ratio and minimal phototoxicity, without averaging. Images were $512 \times 512$ pixels, with a pixel width of $92-110 \mathrm{~nm}$. The experiments were conducted in Tyrode's solution. To block recurrent activity, kynurenic acid $(0.5 \mathrm{~mm})$ was added to the extracellular solution. Action potentials were elicited by passing $50 \mathrm{~mA}$ constant current for $1 \mathrm{~ms}(\sim 50 \%$ above the threshold for eliciting action potentials) through two platinum wires, separated by $\sim 7 \mathrm{~mm}$ and close to the surface of the coverslip.
Fluorescence resonance energy transfer (FRET) determination by acceptor photobleaching was performed as described previously (Laviv et al., 2010). Briefly, the donor (mseCFP) was excited at $440 \mathrm{~nm}$ and its emission was measured at $460-500 \mathrm{~nm}$ before $\left(I_{\mathrm{DA}}\right)$ and after $\left(I_{\mathrm{D}}\right)$ acceptor photobleaching. Excitation was delivered to the acceptor (cpmVen) at $514 \mathrm{~nm}$, and emission was measured at 530-600 nm. Photobleaching of cpmVen was performed with the $514 \mathrm{~nm}$ laser line, by a single point activation module for rapid and efficient multiregion bleaching. The FRET efficiency, $E_{\mathrm{m}}$, was calculated thus:

$$
E m=1-\frac{I_{D A}}{I_{D}}
$$

The ATeam1.03 FRET ratio (FR) was measured for each region of interest (ROI) at four consecutive times: before stimulation, at the completion of stimulation $(10 \mathrm{~Hz}, 90 \mathrm{~s})$, and 3 and $10 \mathrm{~min}$ after its cessation. Intensity of the fluorescence emission excited with the $440 \mathrm{~nm}$ laser was measured simultaneously for mseCFP and cpmVenus at 460-500 nm $\left(I_{\mathrm{D}}\right)$ and $530-600 \mathrm{~nm}\left(I_{\mathrm{A}}\right)$, respectively. Each value was corrected for the background intensity measured from an ROI placed in close proximity to the bouton. In addition, images of the acceptor emission were obtained with the $514 \mathrm{~nm}$ laser excitation before and after stimulation. Only ROIs that exhibited $<15 \%$ change in the intensity of directly excited cpmVen were included in the analysis. Because $40 \%$ of $I_{\mathrm{A}}$ originates from mseCFP, FR was calculated using a correction for the tail of mseCFP's emission as follows:

$$
F R=\frac{I_{A}-0.4 * I_{D}}{I_{D}}
$$

Analysis was performed using custom-written scripts in MATLAB as described previously (Laviv et al., 2010).

An estimation of the change in $[\mathrm{ATP}]_{\text {syn }}$ based on $F R$ was achieved using the Hill equation (Imamura et al., 2009) as follows:

$$
F R=\left(F R_{\max }-F R_{\min }\right) \frac{[A T P]^{n}}{[A T P]^{n}+K_{d}^{n}}+F R_{\min }
$$

The $K_{\mathrm{d}}(0.6 \mathrm{~mm})$ and Hill coefficient $(n=2.1)$ of ATeam1.03 were measured recently in vitro at room temperature (Imamura et al., 2009).

$F R_{\text {min }}$ was measured directly in synapses of neurons treated for $50 \mathrm{~min}$ in ATP-depleting saline, a condition shown previously to reduce [ATP] to near zero levels in active neurons by inhibiting ATP synthesis (Rangaraju et al., 2014). Under these conditions, $F R_{\min }$ was $0.10 \pm 0.02$ (see Fig. $5 B$ ). An identical $F R$ value was obtained when imaging synapses of resting neurons expressing the ATP-insensitive version (SypIATeam $1.03^{\text {R122k/R126K }}$ ) of the synaptic ATP sensor (see above), supporting the conclusion that this floor value represents $F R_{\text {min }}$ in our system.

$F R_{\max }(0.65)$ was calculated using Equation 7 , which was derived from Equation 6 as follows:

$$
F R_{\text {max }}=\left(F R-F R_{\text {min }}\right) \frac{[A T P]^{n}+K_{d}^{n}}{[A T P]^{n}}+F R_{\text {min }}
$$

using as a reference point the reported $[\mathrm{ATP}]_{\mathrm{syn}}$ of resting neurons (1.4 $\mathrm{mM}$, Rangaraju et al., 2014) and the corresponding $F R=0.57 \pm 0.03$ measured in our system.

Finally, the relative change in $[\mathrm{ATP}]_{\text {syn }}$ corresponding to changes in $F R$ was calculated using the following formulae derived from Equation 6:

$$
\begin{gathered}
{[A T P]_{x}=K_{d} \times \sqrt[n]{\frac{F R_{x}-F R_{\text {min }}}{F R_{\text {max }}-F R_{x}}}} \\
\frac{[A T P]_{x}}{[A T P]_{\text {Rest }}}=\sqrt[n]{\frac{F R_{x}-F R_{\text {min }}}{F R_{\text {max }}-F R_{x}} \times \frac{F R_{\text {max }}-F R_{\text {Rest }}}{F R_{\text {Rest }}-F R_{\text {min }}}}
\end{gathered}
$$

\section{Electrophysiology}

Electrophysiological measurements where performed in the whole-cell voltage-clamp configuration at a holding potential of $-70 \mathrm{mV}$ using the EPC-10 amplifier and PatchMaster software (HEKA). Data were sam- 
pled at $10 \mathrm{kHz}$ and filtered at $3 \mathrm{kHz}$. Borosilicate glass pipettes (Sutter Instruments) were pulled using a P-97 horizontal puller (Sutter Instruments) and were fire polished to a resistance of $3-5 \mathrm{M} \Omega$. Recordings were used if the access resistance was $<25 \mathrm{M} \Omega$. Synaptic activity was evoked by inducing action potentials by applying a step depolarization command of $0.5 \mathrm{~ms}$ to $+60 \mathrm{mV}$. Stimulation trains (90 s) were delivered at $10 \mathrm{~Hz}$. Miniature synaptic currents were analyzed using Clampfit (Molecular Devices). Glutamatergic and GABAergic neurons were differentiated by the kinetics of the synaptic currents (Gitler et al., 2004b), which was initially verified by the use of DNQX and picrotoxin, respectively.

\section{Statistics}

Values are reported throughout as mean \pm SEM. Comparisons of two datasets were performed by the Student's $t$ test after confirming a normal distribution by the Shapiro-Wilk normality test or with the Mann-Whitney's nonparametric test otherwise. Multiple comparisons of normally distributed datasets were performed using one-way ANOVA followed by Tukey's post hoc analysis. Comparison of time-dependent processes (FRAP, cumulative charge transfer, frequency facilitation) were performed using repeated-measures ANOVA. Comparison of multiple datasets with distribution that was not deemed normal was performed with the Kruskal-Wallis test followed by Dunn's post hoc test. Significance was set at a confidence level of 0.05 . In all figures, ns denotes $p>0.05,{ }^{*} p<$ $0.05,{ }^{* *} p<0.01$, and ${ }^{* *} p<0.001$. Statistical analysis was performed with Origin, Graphpad Prism, or SPSS 18.

\section{Results}

\section{Intense synaptic activity consumes ATP in the} presynaptic terminal

We hypothesize that $[\mathrm{ATP}]_{\text {syn }}$ modulates the function of synapsin. For this to be an effective control mechanism, $[\mathrm{ATP}]_{\text {syn }}$ should change under physiologically relevant conditions. Because synaptic transmission is a major consumer of cellular energy (MacAskill et al., 2010; Harris et al., 2012), it is reasonable to assume that $[\mathrm{ATP}]_{\text {syn }}$ decreases during intense synaptic activity. Indeed, it was reported recently that $[\mathrm{ATP}]_{\text {syn }}$ drops transiently after the cessation of stimulation that induced extensive exocytosis (Rangaraju et al., 2014) In contrast, in cardiac tissue, local ATP production can offset consumption, leading to steady levels of cytosolic ATP even during rapid pacing (Balaban et al., 1986; Yaniv et al., 2010). In fact, a large fraction of presynaptic terminals contain mitochondria or have one located nearby (Harris et al., 2012), suggesting that the presynaptic terminal is well equipped to answer its own energy needs. Moreover, ATP production by mitochondria is upregulated by increases in cytosolic calcium (Glancy and Balaban, 2012), as may occur during synaptic activity. Therefore, our first aim was to confirm directly whether $[\mathrm{ATP}]_{\text {syn }}$ is altered transiently by intense neuronal activity under the conditions in which synapsin function has been observed. To this end, we used ATeam1.03, an ATP-sensor based on FRET between a monomeric cyan fluorescent protein (mseCFP, the donor) and circularly permutated Venus (cpmVenus, the acceptor) linked by the ATP-binding $\epsilon$-subunit of $\mathrm{B}$. Subtilis F0F1 ATPase (Imamura et al., 2009, and see Fig. 1A). Importantly, the affinity of ATeam 1.03 for ATP lies within the physiological range of ATP concentrations (Gribble et al., 2000). To target ATeam 1.03 to the presynaptic terminal, which is the relevant location for studying the environment of synapsin, we fused it to synaptophysin I (SypI), a transmembranal synaptic vesicle protein (Johnston et al., 1989). The fusion protein SypIATeam1.03 (Fig. $1 A$ ) targeted efficiently to presynaptic terminals of cultured hippocampal neurons (Fig. $1 B$ ), as evidenced by its colocalization with the endogenous v-SNARE synaptobrevin 2 (syb2; Fig. $1 C$ ). A relative measure of $[\mathrm{ATP}]_{\text {syn }}$ in SypIATeam1.03-expressing terminals was obtained by calculating the

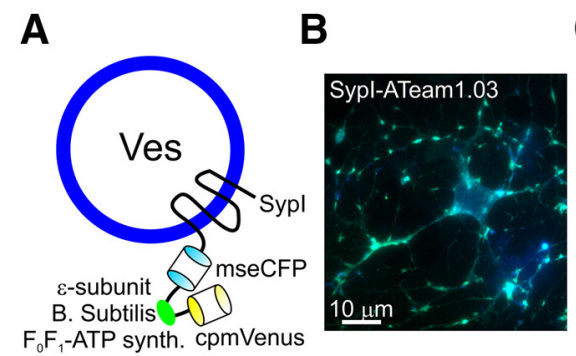

C

D

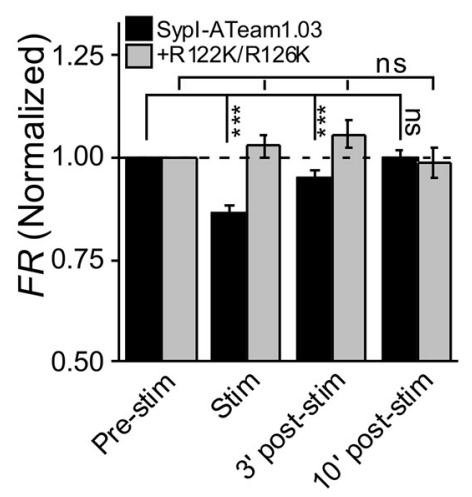

$\mathbf{F}$

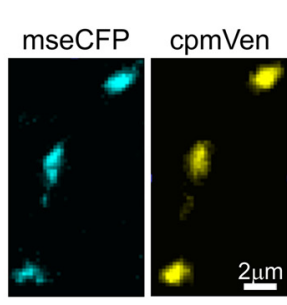

G

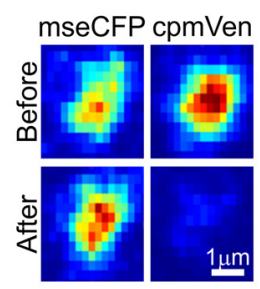

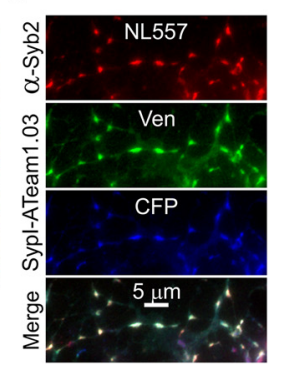

E

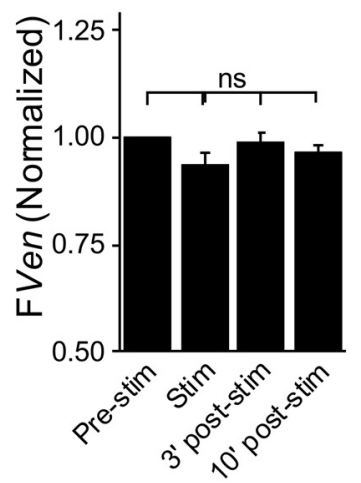

$\mathbf{H}$

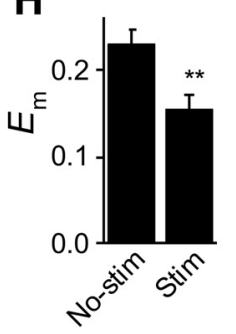

Figure 1. Monitoring activity-dependent changes in $[A T P]_{\text {syn }}$ using a synaptic FRET-based ATP sensor. A, Schematic structure of the synapticly targeted FRET-based ATP sensor SyplATeam1.03. ATeam1.03 was fused to the C-terminal of synaptophysin I, thus targeting it to synaptic vesicles. $\boldsymbol{B}$, Sypl-ATeam1.03 expressed in WT hippocampal neurons. Notice its synaptic localization. C, Sypl-ATeam1.03 expressing neurons were immunostained for synaptobrevin 2 as a marker of synaptic vesicles. Both the mseCFP (blue) and cpmVenus (green) components of Sypl-ATeam1.03 colocalize with endogenous synaptobrevin 2 (red and see merged image at bottom), illustrating that Sypl-ATeam1.03 is targeted to presynaptic terminals. $\boldsymbol{D}$, The SyplATeam1.03 FRET ratio ( $\left.F R, F_{\text {cpmVen }} / F_{\text {msecFP }}\right)$ was calculated in neurons at rest, after $90 \mathrm{~s}$ stimulation at $10 \mathrm{~Hz}$, and finally 3 and $10 \mathrm{~min}$ after cessation of stimulation (black bars). FR was reduced to $82.7 \pm 2 \%$ by stimulation and gradually recovered back to $100 \pm 2 \% 10$ min after cessation of stimulation. Shown are mean \pm SEM values normalized by the initial $F R . n=5 / 84$ coverslips/boutons. Comparisons with prestimulation values were performed by nonparametric Kruskal-Wallis ANOVA, followed by Dunn's post hoc analysis. ${ }^{* * *} p<0.001, \mathrm{~ns}: p>0.05$. Sypl-ATeam1.03 ${ }^{\mathrm{R} 122 \mathrm{~K} / \mathrm{R} 126 \mathrm{~K}}$, which is insensitive to ATP, did not show a significant change in FR after stimulation or during the recovery period (gray bars). $n=4 / 96$. ns: $p>0.05$. $E$, Fluorescence of cpmVen within Sypl-ATeam1.03, elicited directly with the $514 \mathrm{~nm}$ laser line, was not affected by stimulation. $n=1 / 30$. ns: $p>0.05$. $\boldsymbol{F}$, Representative confocal images of boutons of a neuron infected with Sypl-ATeam1.03. G, Pseudocolor-coded fluorescent images of cpmVen (left) and mseCFP (right) in a resting bouton before (top) and after (bottom) cpmVen was bleached. Note the increase in mseCFP fluorescence after cpmVen photobleaching. $\boldsymbol{H}$, FRET efficiency $\left(E_{m}\right)$ of Sypl-ATeam 1.03 in boutons decreased from $0.23 \pm 0.02$ to $0.15 \pm 0.02$ by stimulation. $n=1 / 41$ and $1 / 35$, respectively. Two-tailed unpaired Student's $t$ test. ${ }^{* *} p=$ 0.007 .

FR of cpmVenus and mseCFP when exciting at $440 \mathrm{~nm}$ (Laviv et al., 2010; Fig. 1D). Stimulation ( $10 \mathrm{~Hz}, 90 \mathrm{~s})$ induced a significant drop in FR in the presynaptic terminals. The magnitude of changes in FR do not translate linearly to the effect on $[\mathrm{ATP}]_{\text {syn }}$ 
(Imamura et al., 2009). Therefore, we determined the magnitude of the effect of neuronal stimulation on $[\mathrm{ATP}]_{\mathrm{syn}}$ based on reported resting $[\mathrm{ATP}]_{\text {syn }}$ levels (Rangaraju et al., 2014) and the reported properties of ATeam 1.03 (Imamura et al., 2009; see Materials and Methods for details on calculation). We found that a mean reduction of $13.7 \%$ in $F R$ corresponds to an estimated reduction of $33 \%$ in $[\mathrm{ATP}]_{\mathrm{syn}}$. After cessation of stimulation, $F R$ gradually recovered over $10 \mathrm{~min}$ to prestimulation values (Fig. 1D).

Activity-dependent alterations in the cellular environment, for example, pH changes (Zhang et al., 2010; Svichar et al., 2011; Rangaraju et al., 2014), have the potential to alter the properties of fluorescent proteins. Therefore, the changes in FR that we observed could conceivably reflect environmental effects rather than changes in $[\mathrm{ATP}]_{\text {syn }}$ (Rangaraju et al., 2014). To control for this possibility, we performed similar experiments using the control construct SypI-ATeam1.03 ${ }^{\mathrm{R} 122 \mathrm{~K} / \mathrm{R} 126 \mathrm{~K}}$, in which the $\epsilon$-subunit of the F0F1 ATPase is rendered insensitive to ATP (Imamura et al., 2009). No changes were observed in $F R$ when using this construct (Fig. $1 D$ ), strongly indicating that stimulation does not induce significant ATP-independent alterations in either the donor or acceptor fluorescence. ATeam1.03 incorporates Venus, which is substantially less sensitive to environmental changes than YFP (Nagai et al., 2002). Nevertheless, to address a potential environmental effect on Venus, we excited Venus within ATeam 1.03 directly using the $514 \mathrm{~nm}$ laser line and measured its fluorescence while applying the same stimulation paradigm (Fig. $1 E)$. No significant changes were observed in the fluorescence of Venus, in support of our conclusion that environmental effects do not significantly contribute to the observed changes in $F R$. pH dependence could alternatively be attributed to the $\epsilon$-subunit incorporated into ATeam 1.03. However, we note that, at the reported synaptic ATP concentration (Rangaraju et al., 2014), the sensitivity of ATeam 1.03 to $\mathrm{pH}$ is minimal (see Figure 3 in Imamura et al., 2009).

Finally, we measured FRET using the acceptor-photobleaching method (Laviv et al., 2010), which allows unambiguous determination of FRET efficiency $\left(E_{\mathrm{m}}\right)$ without relying on the fluorescence of the acceptor. Resting terminals exhibited a robust $E_{\mathrm{m}}$ of $0.23 \pm 0.02$ (Fig. $1 F-H$ ), which was reduced by $35 \%$ to $0.15 \pm$ $0.02 \%$ by stimulation (Fig. $1 H$ ), in agreement with the results obtained above using relative FRET imaging. We conclude that $[\mathrm{ATP}]_{\text {syn }}$ drops transiently due to stimulation and recovers over minutes at rest, consistent with the hypothesis that ATP can serve as a modulator of synapsin function.

\section{Mutation of the ATP-binding site does not disrupt the structure of synapsin IIa}

Although it has been shown that all synapsins bind ATP (Esser et al., 1998; Hosaka and Südhof, 1998a, 1998b), the physiological consequences of this fact have not been elucidated to date. In the present study, we focused on ATP binding by synapsin IIa because, in hippocampal glutamatergic neurons, this synapsin isoform has profound effects on synaptic depression (Gitler et al., 2008) and on vesicle clustering (Orenbuch et al., 2012a). Our research strategy was to compare WT synapsin IIa with a synapsin IIa mutant in which ATP binding is abolished. Previous studies have established that a single point mutation in the related synapsin Ia (K269Q) completely abolishes ATP binding without destabilizing the protein structure (Hosaka and Südhof, 1998b). Based on the high homology of the ATP binding residues in synapsins I and II (Hosaka and Südhof, 1998b; Fig. 2A,B) and the phylogenetic conservation of the synapsin $\mathrm{C}$ domain (Kao et al.,
A

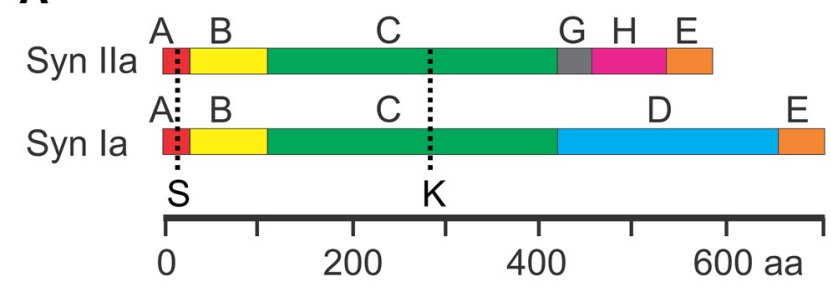

B

SYnII 254-NHREMLTLPTFPVVVKIGHAHSGMGKVKVEN-285 SynI 253-NHKEMLSSTTYPVVVKMGHAHSGMGKVKVDN-284
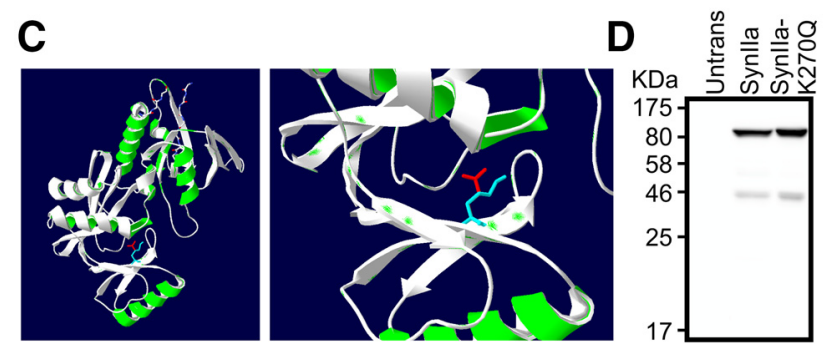

Figure2. Mutating the ATP-binding site in synapsin lla does not overtly alter its structure. $A$, Domain structure of synapsins lla and la. Kmarks the location within the C domain of K270/K269 in synapsins Ila/la, respectively, both of which participate in the coordination of ATP binding. $S$ marks the location of $\mathrm{S10} / \mathrm{S} 9$ in synapsins $l l a / l a$, respectively, which is the consensus PKA/CaMKI phosphorylation site found within domain A. B, Alignment of a 31 aa segment of the well conserved C-domain of synapsin II and synapsin I illustrates the preservation of Lys270/Lys269 (Synll/Synl; underlined and highlighted in yellow) and of its neighboring residues: red, identical amino acid; green, similar polarity. C, Superposition of the crystal structure (PDB-ID: 117N) of the C domain of synapsin II (white ribbon, residues 113-420) and the predicted effect of the $K 2700$ mutation (green ribbon, modeled using SWISS-MODEL). For clarity, only the lysine (K, cyan) and glutamine $(Q$, red) side chains are shown. Left, Whole $C$ domain; right, zoom-in on mutation site. The original and modeled structures overlap. $\boldsymbol{D}$, Western blot (anti synapsin II) of untransfected HEK cells or HEK cells expressing EGFP-Synlla or EGFP-Synlla-K270Q. Both constructs produce a fusion protein of the expected combined molecular weight of synapsin lla and EGFP $(75+28 \mathrm{KDa}$, respectively). A weak lighter band, presumably a degradation product, was present to a similar extent for both constructs.

1999), we introduced the corresponding K270Q mutation into synapsin IIa. Because point mutations can potentially destabilize proteins, we assessed the effect of the mutation on the overall structure of the protein in two manners. First, SWISS-MODEL (Arnold et al., 2006) was used to predict the structure of the mutant using the reported crystal structure of the C-domain of synapsin IIa (PDB-ID: 1I7N) as a template. No obvious structural differences were observed between the WT and predicted structure of the mutant synapsin IIa (Fig. 2C). Second, we expressed EGFP-tagged WT synapsin IIa (EGFP-SynIIa) and the K270Q mutant (EGFP-SynIIa-K270Q) in HEK cells and detected them by Western blot analysis using synapsin II-specific antisera. The products were indistinguishable (Fig. 2C) and their molecular weight was consistent with the fusion of synapsin IIa (Thiel et al., 1990) with EGFP (Prendergast and Mann, 1978). A weak band, which may represent a minor degradation product, appeared to the same extent in both preparations.

Next we investigated whether the mutation affected synaptic targeting of synapsin IIa. Synapsins homodimerize and heterodimerize in vitro and in vivo (Hosaka and Südhof, 1999), a fact that affects their synaptic localization (Gitler et al., 2004a). To avoid interference from endogenous synapsins in measuring synaptic localization and other synaptic properties, all experiments were performed using neurons devoid of synapsins, obtained 
A
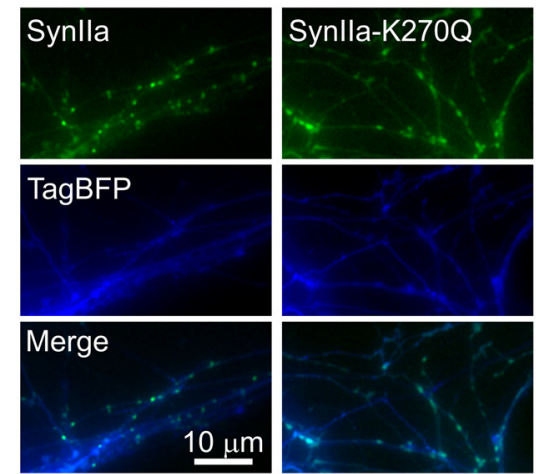

B

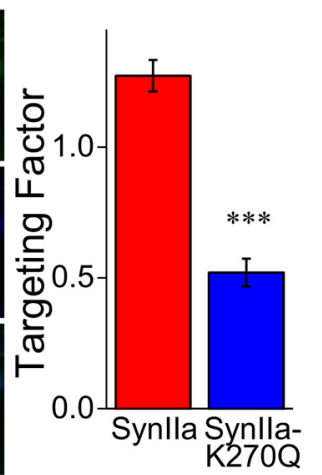

C Ctl Stim Rec
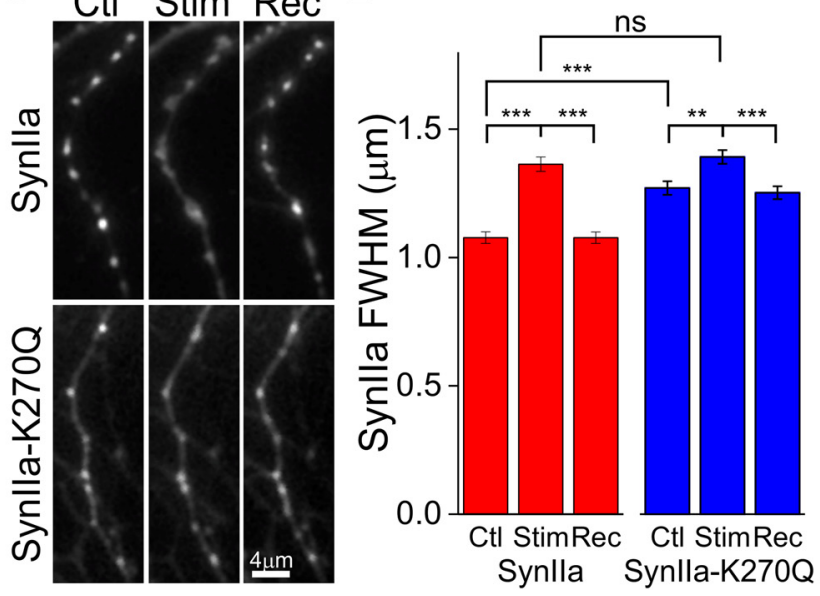

Figure 3. Mutating the ATP-binding site affects the association of synapsin lla with the vesicle cluster. $A$, EGFP-Synlla (left, green) or EGFP-Synlla-K270Q (right) were coexpressed with TagBFP (blue) to assess their synaptic targeting capability. Merged images (bottom) illustrate that both proteins form a punctate pattern along the axons, consistent with synaptic localization. $\boldsymbol{B}$, Synaptic targeting of both proteins was quantified by calculating their "targeting factor" (see Materials and Methods section). Both proteins are enriched in synaptic puncta (i.e., their targeting factor is larger than 0 , one-sample Wilcoxon signed rank test, $p<0.0001$ ), but targeting of the $\mathrm{K} 270 \mathrm{Q}$ mutant is weaker (Mann-Whitney nonparametric test, ${ }^{* * *} p<$ 0.0001). Shown are mean \pm SEM values, $n=19$ and 20 images for EGFP-Synlla and EGFPSynlla-K270Q, respectively, $\sim 20$ synapses analyzed in each. C, EGFP-Synlla and EGFP-SynllaK270Q both form synaptic puncta at rest (Ctl, left), which disperse into the adjacent axonal segments following 1 min of depolarization using hyperkalemic saline (middle). Recovery to the original distribution is achieved after $10 \mathrm{~min}$ in normal saline (right). D, Quantification of $\boldsymbol{C}$. The initial width of EGFP-Synlla-K270Q is larger, but during stimulation, the final width equalizes to that of EGFP-Synlla. The initial width fully recovers after repolarization. $n=24$ and 20 image sets (control, stimulated, recovered) for EGFP-Synlla and EGFP-Synlla-K2700, respectively,

$\sim 20$ matching synapses analyzed per image. Two-way repeated-measures ANOVA followed by Tukey's post hoc analysis. Comparisons within EGFP-Synlla: Ctl versus Stim, ${ }^{* * *} p \ll 0.001$; Stim versus $\operatorname{Rec},{ }^{* *} p \ll 0.001$; Ctl versus $\operatorname{Rec}, p=0.98$. Comparisons within EGFP-SynllaK270Q: Ctl versus Stim, ${ }^{* *} p=0.006$; Stim versus $\operatorname{Rec},{ }^{* * *} p=0.0005$; Ctl versus $\operatorname{Rec}, p=0.61$. Comparison within Ctl: EGFP-Synlla versus EGFP-Synlla-K270Q, ${ }^{* *} p<0.001$; comparison within Stim: EGFP-Synlla versus EGFP-Synlla-K270Q, $p=0.69$.

from synapsin TKO mice (Gitler et al., 2004b). Both EGFPSynIIa and EGFP-SynIIa-K270Q formed a punctate pattern in TKO neurons consistent with synaptic localization (Fig. 3A). This conclusion is supported by their colocalization with syb2 (Fig. 4A). To quantify their propensity to localize in synapses, we measured their targeting index, in which an index of 0 indicates no targeting (see Materials and Methods and Gitler et al., 2004a). Our results confirm that both synapsin IIa and its K270Q mutant target to presynaptic terminals, but indicate that the disruption of
A

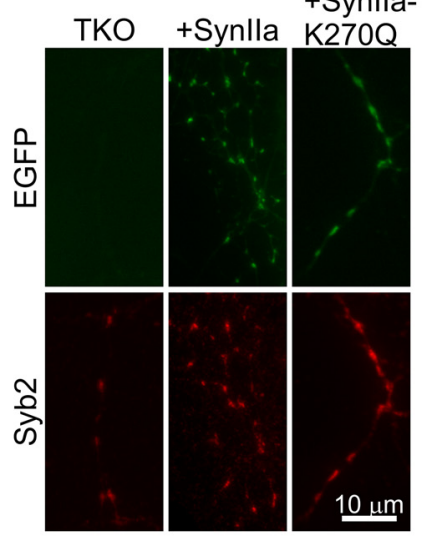

B

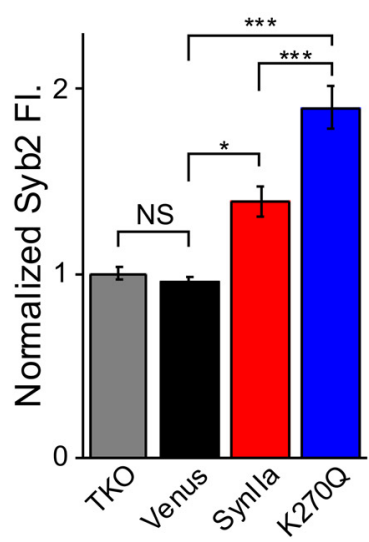

C
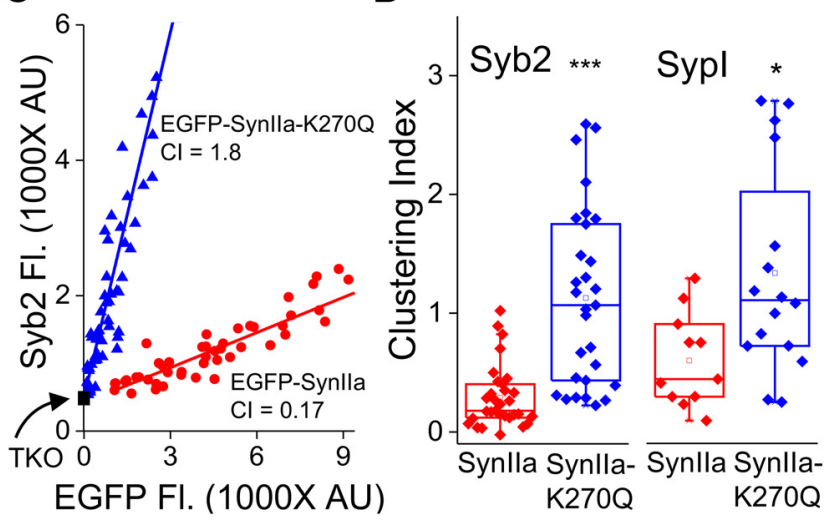

Figure 4. The $\mathrm{K} 270 \mathrm{Q}$ mutation increases the capability of synapsin lla to cluster vesicles at the presynaptic terminal. $A$, TKO neurons were induced to express EGFP-Synlla or EGFP-SynllaK270Q (green) and were subsequently processed for synaptobrevin 2 immunofluorescence (red). $\boldsymbol{B}$, Density of synaptic vesicles within presynaptic terminals was assessed by measuring the synaptobrevin 2 immunofluorescence intensity at the center of mass of the synaptic puncta. The values measured in each imaging session were normalized by the mean values recorded in TKO neurons. Only synapses negative for GAD65 were included in the analysis. Venus, as a control, had no effect on the vesicle density, Synlla increased it, and the K270Q mutant had a significantly larger effect. $n=39,10,30$, and 32 independent images (respectively), each including $>30$ synapses. Values were compared by one-way ANOVA followed by Tukey's post hoc analysis. TKO versus Venus, $p=0.99$; Venus versus Synlla, ${ }^{*} p=0.05$; Venus versus SynllaK270Q, ${ }^{* * *} p<0.001$; Synlla versus Synlla-K270Q, ${ }^{* * *} p=0.0004$. C, Relationship between the synapsin lla content in each synapse and the density of vesicles therein was termed the clustering index (CI). This value was calculated per each image by plotting the syb2 versus EGFP fluorescence intensities and extracting the slope. Shown are representative analyses of single images depicting neurons expressing EGFP-Synlla (red) or EGFP-Synlla-K270Q (blue). D, Left, Distribution of clustering index values calculated for endogenous syb2 in neurons expressing EGFP-Synlla and EGFP-Synlla-K270Q ( $n=25,30$ independent images, respectively). Symbols, Single-image clustering indice; bars, $10-90 \%$ percentiles; box, $25-75 \%$ percentiles; middle bar, median. The clustering index of the K270Q mutant is significantly larger than that of EGFPsynapsin Ila; Mann-Whitney nonparametric test, ${ }^{* * *} p \ll 0.001$. Normality of the distribution was tested using the Shapiro-Wilk test, $p<0.01$. Right, The clustering index values were also calculated for endogenous synaptophysin I. $n=11$ and 16 images, ${ }^{*} p=0.025$. Similarly, the clustering index for the K2700 mutant was larger.

ATP binding by synapsin IIa diminishes its enrichment within this compartment (Fig. 3B). To further examine this finding, we quantified the distribution of synapsin IIa around the presynaptic terminal (Orenbuch et al., 2012b) by measuring the width of the synapsin puncta both at rest and in active terminals (Fig. $3 C, D)$. We found that, at rest, the mutant was distributed more widely around the presynaptic terminals (Fig. 3D). Stimulation has been shown to induce a reversible detachment of synapsin 
from the vesicles and its dispersion into adjacent axonal segments (Chi et al., 2001; Orenbuch et al., 2012b). In agreement, incubation of neurons expressing EGFP-SynIIa in hyperkalemic saline illustrated that synapsin IIa redistributes from the synaptic vesicle cluster into the axon (Fig. 3D). When examining the K270Q mutant, we observed that it too redistributed. The width of the redistributed synapsin was similar for both constructs even though the mutant was already distended at rest. After repolarization, both constructs regained their original distribution (Fig. $3 D$ ). We conclude that ATP binding to synapsin IIa participates in defining its location within the resting presynaptic terminal, but not in its activity-dependent redistribution. The effect of the ATP-binding site mutation on the distribution of the vesicles themselves is addressed below.

\section{Mutating the ATP binding site in synapsin IIa enhances vesicle clustering at the terminal}

In a previous study, we showed that synapsin IIa increases synaptic vesicle density within the presynaptic terminal by enhancing vesicle clustering (Orenbuch et al., 2012a). To observe whether this property is preserved in the synapsin IIa K270Q mutant, we quantified syb2 immunofluorescence intensity in the synaptic puncta of cultured glutamatergic TKO neurons expressing the two synapsin IIa constructs or expressing Venus as a control for infection-related effects (Fig. $4 A$ ). To clarify, "clustering" is used here to describe the specific collection of vesicles within a restricted segment of the axon. In agreement with our previous report, Venus had no effect on syb2 fluorescence intensity, whereas EGFP-SynIIa increased the syb2 signal (Fig. 4B). Surprisingly, not only did the K270Q mutant increase syb2 fluorescence in synaptic puncta, its effect was significantly greater than that of WT synapsin IIa (Fig. 4B). The stronger effect of the K270Q mutant was not due to higher expression levels, because its peak fluorescence intensity in synaptic puncta was actually lower than that exhibited by EGFP-SynIIa ( $62 \pm 6 \%$; mean \pm SEM, $n=22$ and 29 images in 3 independent experiments, respectively, $p<0.001$, Student's $t$ test), in agreement with its wider distribution around the synaptic puncta. This observation implies that negating ATP binding by synapsin IIa promotes vesicle clustering. To explore this finding in greater detail, we plotted the fluorescence of syb2 as a function of EGFP-SynIIa for all individual synapses of each image (Fig. 4C). We found a mostly linear correlation between these two values and, on this basis, we termed the slope of each plot its "clustering index." The clustering indices measured for the K270Q mutant were significantly higher than those of WT synapsin IIa (Fig. 4D, left), in agreement with the suggestion that the K270Q mutant has a higher capacity to cluster vesicles within the presynaptic terminal. An alternative interpretation to our results is that expression of synapsin IIa and of its mutant alters the expression of synaptobrevin 2 or its enrichment in individual synaptic vesicles. To address these possibilities, in addition to syb2, we analyzed the effect of synapsin IIa also on endogenous synaptophysin I. We observed that the K270Q mutation in synapsin IIa enhanced the clustering index measured using SypI antisera in a manner similar to that observed for syb2 (Fig. $4 D$, right). Although both SypI and syb2 are abundant membrane-integral proteins of the synaptic vesicles and are known to interact functionally, they play altogether different roles within the synapse (Kwon and Chapman, 2011; Gordon and Cousin, 2014). Although not conclusive, the congruence of the results obtained using these two proteins implies that the effect of mutating the ATP-binding site in synapsin IIa is indeed mainly related to the density of vesicles within the presynaptic terminal.

\section{ATP binding by synapsin IIa does not affect acute vesicle redistribution}

The aforementioned experiments address the effect of the ATPbinding site on the steady-state vesicle density within resting terminals, but do not explore a possible role for changes in $[\mathrm{ATP}]_{\text {syn }}$ in acute activity-dependent redistribution of vesicles (Kamin et al., 2010). Limited redistribution of SypI has been reported to take place during stimulation of neurons in a manner that could indicate intracellular redistribution of vesicles ( $\mathrm{Li}$ and Murthy, 2001), although this observation has been interpreted to reflect mostly the diffusion of exocytosed synaptophysin within the axonal membrane. To examine directly whether ATP binding to synapsin affects activity-dependent redistribution of vesicles, we imaged SypI-EGFP in neurons that were coinfected with either TagBFP-SynIIa or TagBFP-SynIIa-K270Q (Fig. 5A). We then measured the width of the EGFP-SypI puncta in resting and depolarized neurons. No effect of the ATP-binding site mutation was observed under either condition (Fig. 5A), consistent with similar axonal distribution and similar localization of vesicles within the terminals. Because the interpretation of activitydependent redistribution of SypI is complex, we also investigated whether acute direct depletion of ATP affects the confinement of vesicles within the terminal. ATP depletion was achieved by incubating cultured neurons with deoxy-glucose and oligomycin (ATP-depleting saline), which induce a substantial drop in $[A T P]_{\text {syn }}$ (Rangaraju et al., 2014 and Fig. 5B, top). No effect was observed on the width of SypI-EGFP puncta even after 30 min of incubation (Fig. $5 B$, bottom), inconsistent with macroscopic intracellular redistribution of vesicles. We note that a lack of overt effect on the distribution of vesicles, at the same time that the quantity of vesicles is increased, may indicate a difference in vesicle density. We note that the resolution of wide-field microscopy limits the interpretation of the results presented above, and that future electron microscopy or superresolution studies (Saka and Rizzoli, 2012) may be useful to corroborate our interpretation. We also note a caveat of the pharmacological approach to deplete ATP, which is that other mechanisms that may participate in the determination of vesicular distribution, in addition to synapsin, may have been disrupted.

\section{Vesicle sharing is unaffected in the K270Q synapsin IIa mutant}

Another approach to addressing effects of ATP binding by synapsin IIa on vesicle distribution is to probe how the K270Q mutation affects vesicle mobility (Darcy et al., 2006; Orenbuch et al., 2012a). The idea is that if the K270Q mutation enhances vesicle clustering (Fig. 4) by strengthening the attachment of the vesicles to each other, then this may have a measurable effect on vesicle mobility. We therefore measured intersynaptic vesicle mobility using FRAP of EGFP-tagged synaptophysin I. In these experiments, the fluorescence of SypI-EGFP, which labels all synaptic vesicles, is irreversibly bleached in a single synapse. Because recovery of fluorescence represents the influx of unbleached vesicles from outside the bleached synapse, measuring the extent of fluorescence recovery provides information on vesicle mobility or, in our case, vesicle immobilization (Staras et al., 2010; Staras et al., 2013). Using this technique, we previously reported that synapsin IIa restricts vesicle mobility (Orenbuch et al., 2012a). Here, we compared the effect of TagBFP-labeled WT synapsin IIa and of its K270Q mutant (Fig. 5C) on the recovery of photobleached SypI- 
A

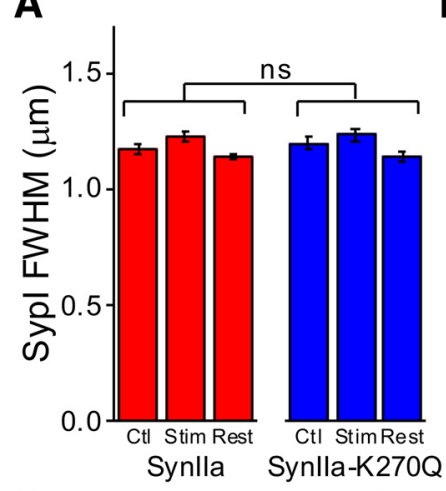

C

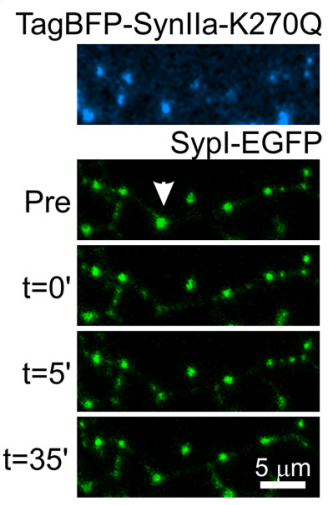

D
B

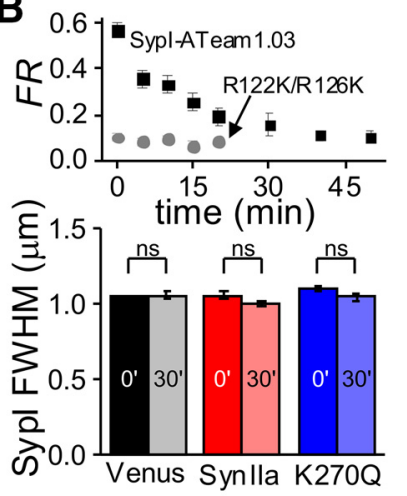

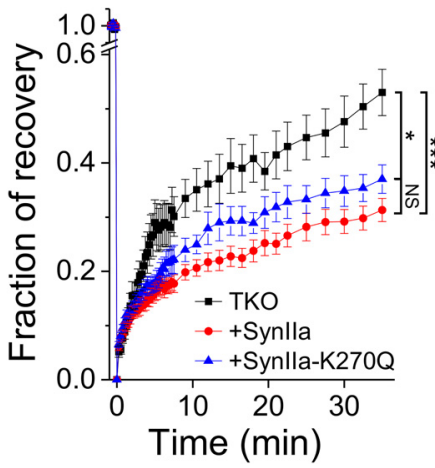

Figure 5. Mutating the ATP-binding site does not affect vesicle redistribution at rest, during synaptic activity, or during depletion of cellular ATP. A, Hyperkalemic depolarization induced widening of Sypl-EGFP puncta in neurons also coexpressing either TagBFP-Synlla or TagBFPSynlla-K270Q. The extent of widening and its recovery were similar in both cases. $n=19$ and 20 image sets (control, stimulated, recovered), respectively, $\sim 20$ matching synapses analyzed per image. Two-way repeated-measures ANOVA, $p=0.76$. $\boldsymbol{B}$, Depletion of ATP does not induce acute redistribution of vesicles. Top, $[A T P]_{\text {syn }}$ was assessed by measuring the acceptor/donor $F R$ of Sypl-ATeam1.03 (black symbols). $n=8$ experiments, $>20$ synapses each. Incubation in ATP-depleting saline induced a progressive decrease in $F R$ down to the level measured using the ATP-insensitive construct Sypl-ATeam 1.03 ${ }^{\text {R122K/R126K }}$ (gray symbols, $n=3$ experiments, $>20$ synapses each). Bottom, The width of Sypl-TagBFP puncta is unchanged after $30 \mathrm{~min}$ of incubation in ATP-depleting saline. $n=15,16$, and 17 image pairs for neurons coexpressing Venus, EGFP-Synlla, or EGFP-Synlla-K270Q, respectively, $\sim 20$ synapses analyzed per pair. Two-way repeated-measures ANOVA followed by Tukey's post hoc analysis, $p=0.12,0.62$, and 0.17 , respectively. C, Vesicle sharing in TKO neurons expressing Sypl-EGFP or those coexpressing Sypl-EGFP and either TagBFP-Synlla or TagBFP-Synlla-K270Q, was assessed by measuring the recovery of Sypl-EGFP after photobleaching single synapses. Shown is a representative experiment in a neuron expressing the TagBFP-Synlla-K270Q. Arrowhead, Bleached synapse. Elapsed time is indicated in minutes, starting with bleaching $\left(t=0^{\prime}\right)$. Sypl-EGFP fluorescence recovered gradually and partially within $35 \mathrm{~min}$. D, Quantification of FRAP experiments illustrating that both TagBFP-Synlla and TagBFP-Synlla-K270Q restricted vesicle sharing in TKO neurons to a similar extent. Recovery was compared by repeated-measures ANOVA, $n=18,33$, and 24 experiments, respectively. TKO versus Synlla, ${ }^{* * *} p=0.001$; TKO versus Synlla-K270Q, ${ }^{*} p=$ 0.048 ; Synlla versus Synlla-K270Q, $p=0.37$.

EGFP (Fig. 5C,D). Both WT synapsin IIa and synapsin IIaK270Q retarded FRAP of SypI-EGFP compared with TKO neurons, but did not differ between them (Fig. 5D), suggesting that both proteins restrict vesicle mobility in a similar manner. We conclude that although ATP binding by synapsin IIa plays an important role in determining the size of the vesicle cluster (Fig. 4 ), it does not regulate the strength of anchoring of the individual vesicles, in agreement with the results presented in the previous section. A different possibility to explain the enhancement of vesicle clustering is that the mutation may increase the number of vesicle-binding opportunities on the cluster without altering the strength of the association itself.

Mutating the ATP-binding site enhances basal evoked synaptic release but does not affect spontaneous release After finding that synapsin IIa enhances vesicle clustering and that excluding ATP from binding to it increases clustering even more (Fig. 4), we examined how both synapsin IIa and its K270Q mutant affect synaptic transmission. It could be anticipated that collecting additional vesicles within the presynaptic terminal would enhance transmission. However, it has been shown previously that basal neurotransmitter release does not correlate with the total amount of vesicles in a terminal (Branco et al., 2010); rather, it is the size of the readily releasable pool (RRP) that is a better predictor of the amplitude of basal release. To examine this point directly, we measured the properties of basal evoked release in infected autaptic neurons. This configuration is especially suited for this because each microisland contains a single neuron, so all inputs impinging upon it are affected by the expression of exogenous proteins. To record basal synaptic properties, autaptic neurons were voltage clamped at $-70 \mathrm{mV}$ and synaptic release was evoked by inducing an action potential in the axonal compartment by a brief step depolarization command (Fig. $6 \mathrm{~A}$ and see Gitler et al., 2004b). The amplitudes of EPSCs recorded in autaptic TKO neurons expressing either EGFP-SynIIa or its K270Q mutant were not different from those recorded in neurons expressing soluble Venus as a control (Fig. 6A, B). Likewise, the time course of the EPSCs was unchanged, as evidenced by the overlap of averaged normalized EPSCs (Fig. 6A). Similarly, the mean amplitude of spontaneous miniature events (mEPSCs) recorded in TKO neurons expressing Venus, EGFP-SynIIa, and EGFP-SynIIa-K270Q were similar $(-20.29 \pm 3.8$ pA, $-20.29 \pm$ $2.1 \mathrm{pA}$, and $-19.87 \pm 2.4 \mathrm{pA}$, respectively, $n=5,9,5$ recordings, 1 -way ANOVA, $p=0.99)$. Nevertheless, we noticed that the amplitude of EPSCs recorded in TKO neurons expressing the K270Q mutant were slightly but significantly larger than those recorded in neurons expressing synapsin IIa (Fig. 6B). A possible explanation for this difference in the EPSC amplitude is an effect on the release probability $\left(P_{\mathrm{r}}\right)$. In fact, it has been suggested that synapsin II suppresses basal release in the diaphragm neuromuscular junction (Coleman et al., 2008). To investigate this possibility in our preparation, we measured frequency facilitation by recording the responses to 5 consecutive stimuli delivered at $10 \mathrm{~Hz}$ (Abramov et al., 2009). Although responses in neurons expressing EGFP-SynIIa exhibited facilitation, EPSCs depressed to a similar extent in neurons expressing the K270Q mutant or in neurons expressing Venus (Fig. 6C,D). This result implies (but does not prove) that synapsin IIa decreases vesicular $P_{\mathrm{r}}$, as previously suggested, and, moreover, that the K270Q mutation obviates this effect. On the basis of our collective results, we speculate that synapsin IIa, and more so its mutant, increase the size of the total vesicle population and consequently the size of the RRP, an effect that could lead to enhanced basal release. However, in parallel, synapsin IIa decreases the per-vesicle $P_{\mathrm{r}}$, an effect that is not supported by the K270Q mutant. As an end result, basal release in neurons expressing synapsin IIa remains unchanged compared with control neurons, but is lower than that observed in neurons expressing the K270Q mutant.

\section{ATP binding by synapsin IIa is essential for its ability to} sustain synaptic release during periods of intense activity Synaptic depression is a progressive and reversible, activitydependent decrease in synaptic transmission that is typically observed during sustained high-frequency stimulation. One of its suggested mechanisms, although not the sole one, is the gradual depletion of vesicles in the RRP (Zucker and Regehr, 2002; Re- 
A
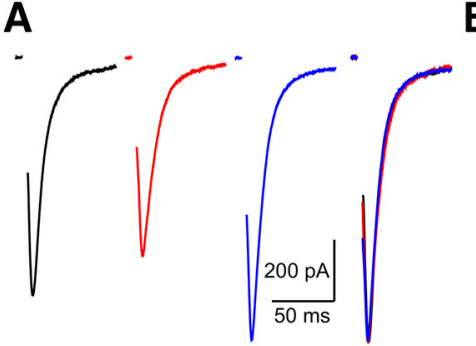

Venus +Synlla +Synlla- Norm. K270Q

C

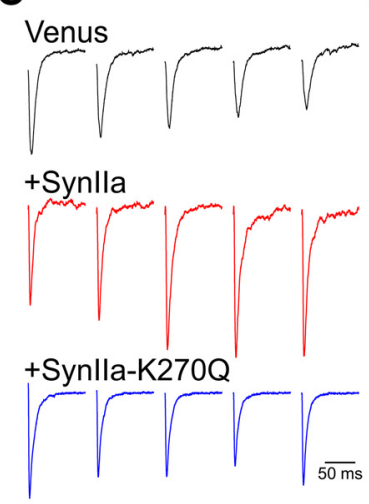

D

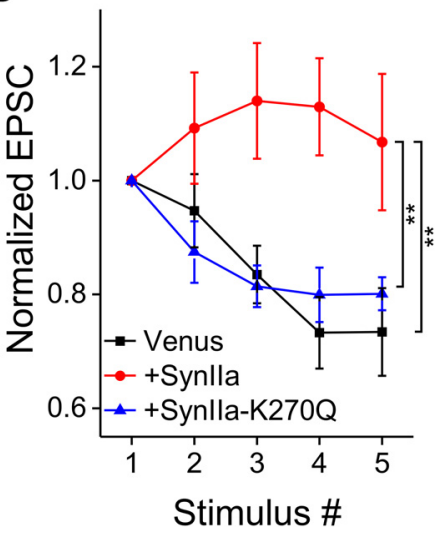

Figure 6. Effect of synapsin lla and its mutant on basal synaptic properties. A, Averaged EPSCs elicited by brief depolarization commands in autaptic synapsin TKO neurons. Shown are control TKO neurons expressing Venus (black, $n=14$ ), EGFP-Synlla (red, $n=15$ ), and EGFPSynlla-K270Q (blue, $n=22$ ). The action potential current preceding the EPSC was blanked for the purpose of clarity. Superposition of averaged normalized EPSCs (right traces) illustrates that they fully overlap. $\boldsymbol{B}$, Quantification of mean EPSC amplitudes. Mean \pm SEM, $848 \pm 7.6 \mathrm{pA}$, $743 \pm 7.1 \mathrm{pA}$, and $1092 \pm 12.3 \mathrm{pA}, n=14,18$, and 22, respectively. Notice that both Synlla and its $\mathrm{K} 270 \mathrm{Q}$ mutant do not differ from control, although they do differ from each other. One-way ANOVA followed by Tukey's post hoc analysis: control versus EGFP-Synlla, $p=0.78$; control versus EGFP-Synlla-K270Q, $p=0$. 24; EGFP-Synlla versus EGFP-Synlla-K270Q, ${ }^{*} p=$ 0.038. C, The K270Q mutation reduces the effect of synapsin lla on frequency modulation of release. Neurons were stimulated at $10 \mathrm{~Hz}$ and the responses to five subsequent stimuli were monitored. Shown are representative recordings, as indicated. Control neurons (Venus) and neurons expressing EGFP-Synlla-K270Q exhibited depression, whereas neurons expressing EGFP-Synlla exhibited facilitation. $\boldsymbol{D}$, Quantification of frequency-modulation. $n=11,8$, and 11 , respectively. Repeated-measures ANOVA followed by Tukey's post hoc analysis. Control versus EGFP-Synlla, ${ }^{* *} p=0.006$; control versus EGFP-Synlla-K270Q, $p=0.99$; EGFP-Synlla versus EGFP-Synlla-K270Q, ${ }^{* *} p=0.008$.

gehr, 2012). Therefore, the rate of depression is influenced both by changes in the rate of exocytosis and by the resupply of releasable vesicles (Gabriel et al., 2011). Due to the key role that the synapsins play in managing vesicle reserves, most manipulations affecting the expression of the synapsins (either deletion or overexpression) typically result in alteration of the kinetics of synaptic depression (acceleration or deceleration, respectively). In glutamatergic hippocampal neurons, synapsin IIa plays a key role in defining depression kinetics. Moreover, it is the only synapsin isoform to rescue the synaptic depression phenotype of the synapsin TKO mouse; it even significantly slows synaptic depression in glutamatergic autaptic neurons of wild-type mice, in contrast to synapsin I, which had no effect (Gitler et al., 2008). To explore the role that ATP binding to synapsin IIa plays in sustaining synaptic transmission during periods of intense activity, we induced short-term depression in autaptic TKO hippocampal glutamatergic neurons expressing Venus, synapsin IIa, or its K270Q
A
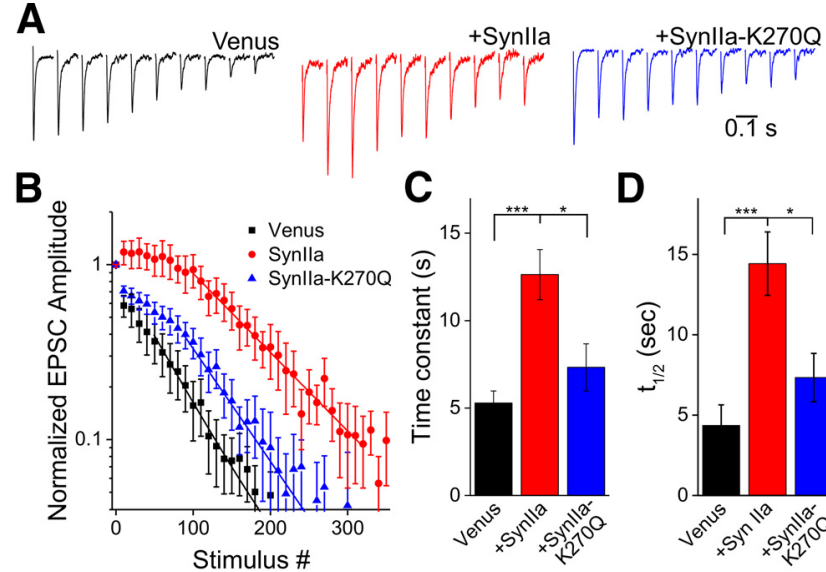

D
E

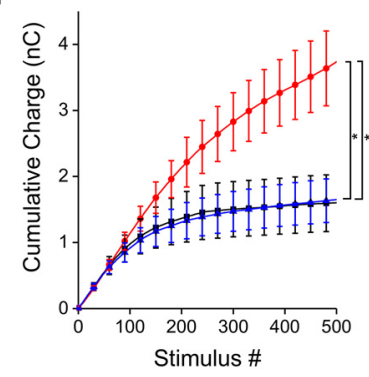

$\mathbf{F}$

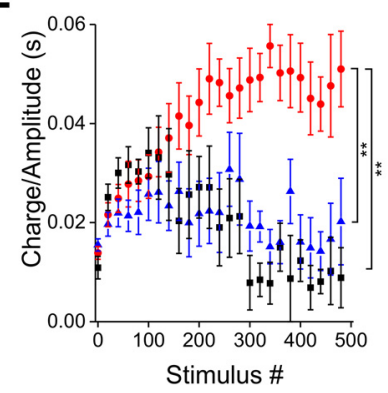

Figure 7. Mutating the ATP-binding site annuls the effect of synapsin lla on synaptic depression. $\boldsymbol{A}$, Neurons were stimulated 900 times at $10 \mathrm{~Hz}$ and their synaptic responses were recorded. Shown are representative responses for control neurons expressing Venus (control, black) and for TKO neurons expressing EGFP-Synlla (red) or its K270Q mutant (blue). Every $15^{\text {th }}$ response in the stimulation train is shown. $\boldsymbol{B}$, Normalized end-point subtracted semilog plots of mean \pm SEM EPSC responses to stimulation trains as in $\boldsymbol{A}$ ( $n=11,7$, and 11 , respectively). Depression is deferred by the expression of EGFP-Synlla, but not of EGFP-Synlla-K270Q, compared with the Venus control. Furthermore, the rate of depression, once initiated, is slower for EGFP-Synlla than for the K2700 mutant. For clarity, symbols and error bars are shown for every $10^{\text {th }}$ stimulus, and down to $\sim 5 \%$ of the initial amplitude. Solid lines denote single-exponent fits applied starting 5, 10, and 9 s after the first EPSC, respectively. C, Average time constant of synaptic depression, calculated by fitting the EPSC amplitudes with a single-exponent decay function as shown in $\boldsymbol{B}$. One-way ANOVA followed by Tukey's post hoc analysis. Control versus EGFP-Synlla, ${ }^{* * *} p=0.0008$; control versus EGFP-Synlla-K270Q, $p=0.39$; EGFP-Synlla versus EGFP-Synlla-K270Q, ${ }^{*} p=0.014$. D, Because synaptic depression follows complex kinetics, the $t_{1 / 2}$ of depression was calculated. EGFP-Synlla delays depression, whereas the K2700 mutant does not. One-way ANOVA followed by Tukey's post hoc analysis. Control versus EGFP-Synlla, ${ }^{* * *} p=0.0005$; control versus EGFP-Synlla-K270Q, $p=0.32$; EGFP-Synlla versus EGFP-Synlla$\mathrm{K} 270 Q,{ }^{*} p=0.013$. $E$, Cumulative charge transfer during EPSC trains. Although EGFP-Synlla significantly increased the charge transfer throughout the train, the K270Q mutant was ineffective in this respect. $n=6,6$, and 8 , respectively. Repeated-measures ANOVA using every fifth EPSC value within the $200^{\text {th }}-500^{\text {th }}$ stimuli range, followed by Tukey's post hoc analysis. Control versus EGFP-Synlla, ${ }^{*} p<0.049$; control versus EGFP-Synlla-K270Q, $p=0.99$; EGFPSynlla versus EGFP-Synlla-K270Q, ${ }^{*} p=0.033$. F, Synapsin lla progressively increases the ratio between the charge and amplitude of each EPSC during stimulation trains, consistent with a gradual desynchronization of synaptic release. The K2700 mutation annuls this effect. Repeated-measures ANOVA, followed by Tukey's post hoc analysis. Control versus EGFP-Synlla, ${ }^{* *} p=0.002$; control versus EGFP-Synlla-K270Q, $p=0.92$; EGFP-Synlla versus EGFP-Synlla$\mathrm{K} 270 \mathrm{Q},{ }^{* *} p=0.006$.

mutant. Synaptic depression did not exhibit single-exponential kinetics throughout its progression (Fig. $7 A, B$ ), consistent with a multiple-pool model of activity-dependent vesicle dynamics exhibiting supply-rate depression (García-Pérez et al., 2008; Gabriel et al., 2011). Therefore, we quantified its time course using two complementary methods. The first was to measure the time constant (slope) of the late linear segment that is observed in a semilogarithmic plot of synaptic depression (Fig. 7C) and is 
probably limited by vesicle recruitment (Neher, 2012), whereas the second was based on measuring the time that elapses from the beginning of stimulation until the EPSC amplitude reached half its initial size (Fig. 7D). As expected, expression of EGFP-SynIIa significantly slowed synaptic depression according to both criteria compared with control neurons expressing Venus (Fig. 7A$D$ ). In fact, synaptic transmission initially facilitated (see also Fig. 6), was enhanced for $\sim 100$ stimuli, and only then depression was observed. Conversely, EGFP-SynIIa-K270Q did not affect the kinetics of synaptic depression (Fig. $7 A-D$ ). Because the K270Q mutation obviates ATP binding by synapsin IIa, we conclude that ATP binding plays a key role in controlling the kinetics of synaptic depression. An approach to assessing total release during a train is to integrate the EPSC traces (also termed the charge transfer). We observed that the charge transfer was significantly enhanced by synapsin IIa, but not by the K270Q mutant (Fig. 7E), in agreement with the specific slowing of synaptic depression by synapsin IIa. Recently, it was reported that synapsin II progressively desynchronizes synaptic release during trains of stimuli in GABAergic neurons (Medrihan et al., 2013), an effect that can increase the charge transfer during a train of stimulation over and above the observed effect on the amplitude (Kaeser and Regehr, 2014). We measured the ratio between the charge and the amplitude of the EPSCs throughout the train to assess whether response desynchronization may contribute to the observed effect on the charge transfer. We found that synapsin IIa, but not the K270Q mutant, increased the charge to amplitude ratio, consistent with a gradual desynchronization of synaptic release (Fig. $7 F$ ). Altogether, our results indicate that mutating the ATPbinding site in synapsin IIa results in two contrasting outcomes: although more vesicles are contained within the presynaptic terminal expressing the mutant compared with WT synapsin IIa (Fig. $4 B, D$ ), these terminals are less capable of supporting sustained synaptic release (Fig. 7).

\section{Interaction between ATP binding by synapsin IIa and its phosphorylation state}

One of the best documented mechanisms for the regulation of the synapsins is their phosphorylation. Synapsin IIa has in its A domain a well conserved serine (S10; Fig. $2 A$ ) that serves as a phosphorylation site for both protein kinase A (PKA) and calcium/ calmodulin-dependent kinase I (CaMKI) (Cesca et al., 2010). This site is homologous to $S 9$ in synapsin I, which has been identified as a key activity-dependent regulator of the association of vesicles with synapsin (Hosaka et al., 1999; Menegon et al., 2006). To determine whether interfering with ATP binding to synapsin IIa affects phosphorylation at S10, we used an antibody that specifically identifies phospho-S10. Although this antibody was originally raised against phospho-S9 in synapsin I, we found that it also identifies phospho-S10 in synapsin IIa, probably due to the high homology of the protein sequence flanking this site (Fig. $8 A$ ). To demonstrate the utility of this antibody, we immunostained TKO neurons expressing exogenous TagBFP-SynIIa under conditions expected to induce or repress phosphorylation at S10. Indeed, synaptic terminals in neurons depolarized by hyperkalemic saline were extensively stained, whereas those in resting neurons treated with $\mathrm{H} 89(10 \mu \mathrm{M})$, an inhibitor of PKA, exhibited much weaker staining (Fig. 8B). Because the absolute peak intensity of the synapsin IIa signal in synapses is affected by the K270Q mutation (see above), we normalized the phospho-S10 immunofluorescence signal in each synapse by the TagBFP signal, producing a phosphorylation ratio that is independent of the synapsin IIa quantity. The phosphorylation ratio measured in the presence
A

SYnII 1 MMNFLRRRLSDSSFIANLPNGYMTDLQRP-29 SYnI 1 -MNYLRRRLSDSNFMANLPNGYMTDLQRP-28
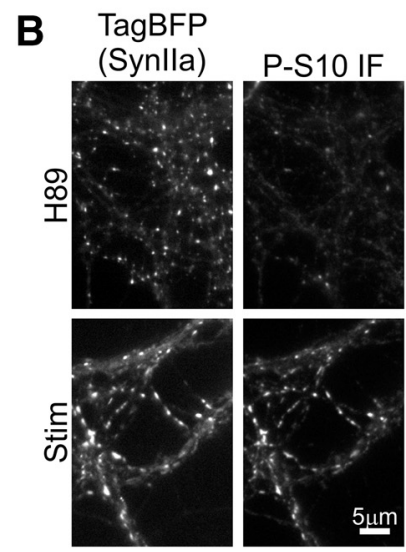

C
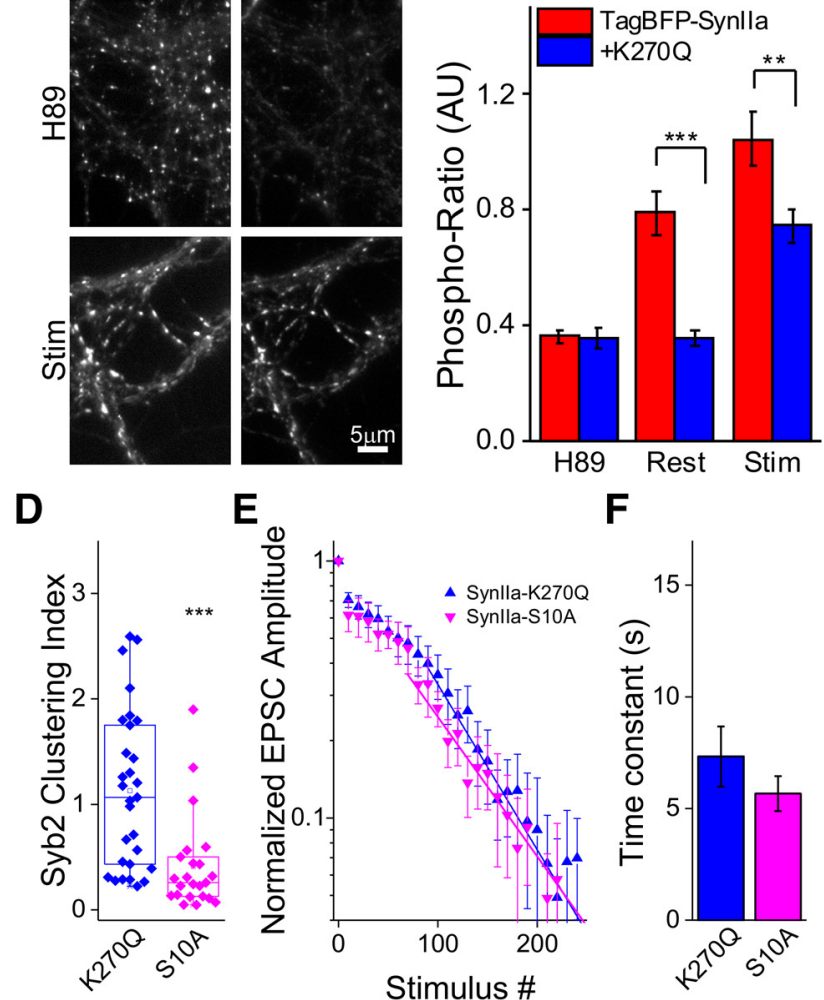

$\mathbf{F}$

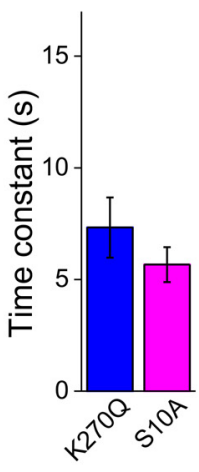

Figure 8. Mutating the ATP-binding site in the C domain of synapsin lla attenuates phosphorylation in site 1 within the A domain. $\boldsymbol{A}$, Sequence homology of the A domains of synapsin Il and synapsin I. Red, Identities; yellow background, phosphorylation site 1. B, An antibody for phosphorylation site $1(\mathrm{~S} 9)$ in synapsin I interacts specifically with the homologous site in synapsin Ila (S10). Neurons from synapsin TKO mice, which lack all synapsins, were induced to express TagBFP-Synlla (left column) and were immunostained for phospho-S10 (IF P-S10, right column). Neurons treated with H89 (top row) exhibited background immunofluorescence, whereas depolarized neurons (bottom row) exhibited robust P-S10 immunofluorescence. $\boldsymbol{C}$, Comparison of the extent of phosphorylation of TagBFP-Synlla and its K2700 mutant. Although the phosphorylation ratio in $\mathrm{H} 89$-treated samples was similar, it was significantly lower for the $\mathrm{K} 2700$ mutant both in resting and in stimulated neurons. $n=21,28,26,29,30$, and 32 independent images per condition, respectively, per column from left to right, one-way ANOVA followed by Tukey's post hoc analysis comparing TagBFP-Synlla versus TagBFP-Synlla-K270Q in $\mathrm{H} 89, p=0.11$; the same in resting neurons, ${ }^{* * *} p \ll 0.001$; and the same in stimulated neurons, ${ }^{* *} p=0.004$. D, Clustering index measured using immunolabeling of endogenous syb2 is significantly lower for EGFP-Synlla-S10A than it is for EGFP-Synlla-K270Q. $n=29$ and 23 images for $\mathrm{K} 270 \mathrm{Q}$ and $\mathrm{S} 10 \mathrm{~A}$, respectively, $p=0.0001$, Mann-Whitney nonparametric test. $\boldsymbol{E}$, Semilogarithmic end-point subtracted plots of the mean normalized EPSC amplitudes elicited by $10 \mathrm{~Hz}$ stimulation in glutamatergic autaptic neurons expressing EGFP-Synlla-K270Q ( $n=$ 11) or EGFP-Synlla-S10A $(n=5)$. For clarity, symbols and error bars are shown for every $10^{\text {th }}$ stimulus and down to $\sim 5 \%$ of the initial amplitude. Solid lines denote single-exponent fits applied in the indicated range. Synaptic responses depressed in a similar manner in both groups. $\boldsymbol{F}$, Average time constant of synaptic depression for data shown in $\boldsymbol{E}$. $p=0.44$, Student's $t$ test.

of H89 was similar for neurons expressing synapsin IIa and its K270Q mutant (Fig. 8C). In the case of TagBFP-SynIIa, the phosphorylation ratio was higher in resting cultures that were not treated with $\mathrm{H} 89$, suggesting the existence of basal phosphorylation activity in resting cultures, as was observed previously ( $\mathrm{Me}-$ negon et al., 2006). This was not observed for the K270Q mutant. Finally, depolarization of the neurons by hyperkalemic saline in- 
duced a significant increase in the previously-mentioned ratio for both conditions, but more so for TagBFP-SynIIa (Fig. 8C).

The fact that the K270Q mutation reduced the magnitude of phosphorylation of synapsin IIa at the S10 residue implies an interaction between these two distant sites. To separate between the effects of ATP binding and S10 phosphorylation on the properties of synapsin IIa, we produced a S10A mutant of synapsin IIa that is unphosphorylatable at $\mathrm{S10}$. We then measured its effect on the two main parameters that were altered by the K270Q mutation (Fig. $8 D-F$ ). We found that the S10A mutant was less efficient than the K270Q mutant in clustering vesicles (Fig. 8D), whereas it was similar to wild-type synapsin IIa (cf. Fig. $4 D$ ). We therefore suggest that, at rest, the K270Q mutant affects vesicle distribution in terminals independently of its dampening effect on the phosphorylation of S10. Conversely, the S10A mutant was as ineffective as the $\mathrm{K} 270 \mathrm{Q}$ mutant in rescuing synaptic depression in active neurons (cf. Figs. $8 E, F, 7 B, C$ ). Although it is possible that the two mutations disrupt independently the capability of synapsin IIa to rescue synaptic depression, it is also just as likely that the effect of the K270Q mutation on synaptic depression is mediated by its inhibitory effect on the phosphorylation of S10.

\section{Discussion}

Although the fact that synapsins are ATP-binding proteins was reported more than a decade ago, the implications of this observation have not been explored. Fortunately, the amino acids that participate in ATP binding to synapsin I were mapped (Esser et al., 1998; Hosaka and Südhof, 1998b), allowing us to exploit the high homology of the C-domains of synapsin I and II to identify the phylogenetically conserved K270 residue in synapsin IIa (Fig. 2). We mutated K270 to glutamine and examined its effect on key presynaptic properties. Our results indicate that preventing ATP binding to synapsin IIa increases its capability to cluster vesicles, attestable by the higher syb2 content in synaptic puncta of neurons expressing SynIIa-K270Q and by the larger vesicle clustering index measured using both syb2 and sypI (Fig. 4). The mutation had no measurable effect on intersynaptic vesicle mobility (Fig. 5), indicating that it was not necessarily the strength of the association between synapsin IIa and vesicles that is enhanced, but rather the quantity of per-vesicle effective association sites. Nevertheless, the mutation lowered the capacity of synapsin IIa to localize to the synaptic terminals (Fig. 3) or to sustain synaptic transmission during periods of intense demand (Fig. 7). This probably reflects a disruption of the synapsin-dependent recruitment of reserve vesicles to the RRP that takes place during periods of intense activity (García-Pérez et al., 2008; Gabriel et al., 2011; Neher, 2012; Walter et al., 2013), with only a minor effect on the resting size of the RRP (Fig. 6 and Gitler et al., 2008). Therefore, the ATP-binding site mutation appears to produce a deficit in the coupling between activity (demand) and supply of the stored vesicles; phosphorylation of synapsin IIa could serve as the underlying mechanism.

Synapsins are known to control vesicle availability by providing reserve vesicles during periods of intense synaptic activity (Cesca et al., 2010), but were also shown in some cases to affect basal release (Llinás et al., 1985; Hackett et al., 1990; Hilfiker et al., 1998; Hilfiker et al., 2005; Coleman et al., 2008). The supply of vesicles involves the phosphorylation of synapsins, which profoundly lowers their affinity to vesicles and to cytoskeletal elements (Hosaka et al., 1999; Cesca et al., 2010; Bykhovskaia, 2011). We found that the K270Q mutation diminishes the phosphorylation of synapsin on its PKA/CaMKI phosphorylation site 1 (Fig. 8). This key observation implies a functional interaction between the ATP-binding site and the regulation of synapsin IIa by phosphorylation, even though the two sites are quite distant within the protein structure (Fig. 2A). Although we did not identify how ATP binding alters the phosphorylation of synapsin IIa, we note that synapsin has been proposed to act as a phosphate transfer enzyme (Esser et al., 1998; Hosaka and Südhof, 1998b). Because its target was not identified, it is possible that synapsin IIa directly or indirectly enhances its own phosphorylation. To determine whether the effects of the K270Q mutation on vesicle clustering and on synaptic depression are mediated by its observed inhibition of phosphorylation at S10, we examined the effect of the S10A mutation directly on both properties (Fig. $8 D-F$ ). We found that the effect of $\mathrm{K} 270 \mathrm{Q}$ on vesicle clustering in resting neurons is independent of S10, because it is not recapitulated by the S10A mutation (Fig. 8D). In contrast, both the K270Q and S10A mutations disrupted the capability of synapsin IIa to slow synaptic depression in active neurons (Fig. 8E,F). Our results indicate that synapsin IIa is regulated both by $[\mathrm{ATP}]_{\mathrm{syn}}$ and by phosphorylation. We postulate that ATP serves as an allosteric modulator of synapsin IIa, as was shown for other proteins (Henquin, 2000; Marchetti et al., 2013; Stölting et al., 2013), and predict that ATP-free synapsin IIa should be more affine to vesicles because it is the $\mathrm{K} 270 \mathrm{Q}$ mutant, and not $\mathrm{S} 10 \mathrm{~A}$, that clusters vesicles to a higher degree. This prediction remains to be corroborated directly by biochemical assays. Furthermore, we propose that the deficit of the SynIIa-K270Q mutant in rescuing synaptic depression is due to its lower propensity for phosphorylation at S10.

Although this is not necessarily the only interpretation of our results, we suggest that, in this manner, synapsin IIa fine-tunes the processes of vesicle clustering and vesicle recruitment, depending on the level and duration of neuronal activity. We speculate that, at rest, dephosphorylated synapsin IIa tends to accumulate and cluster vesicles in the reserve pool. Mutating the ATP-binding site enhances this function due to the proposed higher affinity of synapsin IIa to vesicles when not bound to ATP. With the commencement of activity, phosphorylation of synapsin IIa facilitates the recruitment of the previously accumulated vesicles. Both K270Q and S10A are deficient in this respect due to their lower/nonaccessibility to phosphorylation at S10. Finally, we predict that the decrease in $[\mathrm{ATP}]_{\mathrm{syn}}$ associated with sustained activity eventually enhances the affinity of synapsin IIa to vesicles, impeding the severe depletion of remaining vesicles.

The idea that $[\mathrm{ATP}]_{\text {syn }}$ modulates synapsin function has merit mostly if $[\mathrm{ATP}]_{\mathrm{syn}}$ is affected by the activity level of the neuron. Because consumption and production of ATP in cardiomyocites are well matched under many physiological conditions, steady levels are achieved (Balaban et al., 1986; Yaniv et al., 2010). In part, this is due to the tight regulation that is exerted by ATP, its precursors, and its metabolites on cellular metabolism. Conversely, intracellular ATP concentration is known to vary in other cell types, such as pancreatic $\beta$-cells; in those cells, ATP fluctuates as part of the mechanism of insulin secretion (Henquin, 2000). Various steps of neuronal activity have been shown to be major energy sinks (Harris et al., 2012), leading to the question of whether ATP fluctuates in an activity-dependent manner in synapses. Changes in ATP were indeed documented during pathological epileptiform activity in hippocampal cultured slices (Toloe et al., 2014). Recently, the specific steps of neurotransmission that consume most ATP were visualized under more physiological activity levels using the synaptically targeted modified luciferase termed Syn-ATP (Rangaraju et al., 2014). This study concluded that $[\mathrm{ATP}]_{\text {syn }}$ decreases minutes after completing a 
period of strong stimulation, during the time when vesicle recycling occurs. In contrast, we recorded a decrease in $[\mathrm{ATP}]_{\mathrm{syn}}$ immediately after delivering stimulation at $10 \mathrm{~Hz}$; this decrease recovered over several minutes (Fig. 1D). Because we did not measure $[\mathrm{ATP}]_{\mathrm{syn}}$ during the stimulation train itself, we cannot define the precise timing of the drop in $[\mathrm{ATP}]_{\mathrm{syn}}$, but it appears to take place earlier than reported. A possible reason for this apparent discrepancy is that the underlying principle of action of SynATP and SypI-ATeam1.03 are dissimilar; SypI-ATeam 1.03 is based on FRET, whereas Syn-ATP relies on enzymatic activity. Therefore, key biochemical properties of the probes may differ in a manner that can affect their output. For example, they may be differently affine to ATP complexed with either magnesium or calcium ions (Wilson and Chin, 1991), especially considering that the sensitivity of the binding constants of ATP to these divalent ions depends on $\mathrm{pH}$ (Schoenmakers et al., 1992). In addition, the sensitivity of the probes themselves to $\mathrm{pH}$ may differ; this parameter was reported to be minimal for ATeam 1.03 at the level of ATP concentrations that is relevant to our study (see Fig. 3 in Imamura et al., 2009). Notwithstanding the observed differences, it is clear that synaptic activity alters $[\mathrm{ATP}]_{\text {syn }}$ within a time frame relevant to synapsin function, lending credibility to the claim that $[\mathrm{ATP}]_{\text {syn }}$ can serve as a modulator of synapsin. This conclusion apparently contrasts with findings obtained using permeabilized cells showing that ATP is not essential for mobilization of vesicles specifically from the recycling pool (Mozhayeva et al., 2004).

Are the changes in $[\mathrm{ATP}]_{\text {syn }}$ within a relevant range to affect synapsin? The in vitro affinity of synapsin to the ATP analog ATP $\gamma S$ is in the submicromolar range (Hosaka and Südhof, 1998b; Brautigam et al., 2004), which is outside of the physiologically relevant range. However, it was shown that $\mathrm{Mg}^{2+}$ shifts this affinity to the low millimolar range (Hosaka and Südhof, 1998b), placing it within the range in which activity-related $[\mathrm{ATP}]_{\text {syn }}$ changes were reported (Rangaraju et al., 2014) and potentially maximizing the projected sensitivity of synapsin to changes in $[\mathrm{ATP}]_{\text {syn }}$. We note that, unlike synapsin II, ATP binding to synapsin I is calcium dependent (Hosaka and Südhof, 1998b). Because persistent synaptic activity induces temporary increases in cytosolic calcium, the putative regulation of synapsin I by ATP may be more strongly dependent on the activity level of the neuron than on resultant alterations in $[\mathrm{ATP}]_{\mathrm{syn}}$. The study of the differential consequences of ATP binding to synapsin I and II is therefore warranted. It is important to realize that the interplay between activity-dependent changes in calcium, magnesium, $\mathrm{pH}$, and $[\mathrm{ATP}]_{\mathrm{syn}}$, as well as in the affinity of synapsins to ATP, are expected to be more complex than those alluded to here. Future quantitative models on the effects of $[\mathrm{ATP}]_{\mathrm{syn}}$ on synapsin-related vesicle availability will need to take this fact into account.

The regulation of synaptic transmission is a complex and fascinating subject. Here, we add another twist to this already convoluted story by revealing that the properties of synapsin IIa, a protein that affects the availability of vesicles during intense synaptic activity, is tightly bound to the presynaptic dynamics of ATP, a subject that has recently come into focus.

\section{References}

Abbott LF, RegehrWG (2004) Synaptic computation. Nature 431:796-803. CrossRef Medline

Abramov E, Dolev I, Fogel H, Ciccotosto GD, Ruff E, Slutsky I (2009) Amyloid-beta as a positive endogenous regulator of release probability at hippocampal synapses. Nat Neurosci 12:1567-1576. CrossRef Medline

Arnold K, Bordoli L, Kopp J, Schwede T (2006) The SWISS-MODEL work- space: a web-based environment for protein structure homology modelling. Bioinformatics 22:195-201. CrossRef Medline

Balaban RS, Kantor HL, Katz LA, Briggs RW (1986) Relation between work and phosphate metabolite in the in vivo paced mammalian heart. Science 232:1121-1123. CrossRef Medline

Baldelli P, Fassio A, Valtorta F, Benfenati F (2007) Lack of synapsin I reduces the readily releasable pool of synaptic vesicles at central inhibitory synapses. J Neurosci 27:13520-13531. CrossRef Medline

Bergsman JB, Krueger SR, Fitzsimonds RM (2006) Automated criteriabased selection and analysis of fluorescent synaptic puncta. J Neurosci Methods 152:32-39. CrossRef Medline

Boido D, Farisello P, Cesca F, Ferrea E, Valtorta F, Benfenati F, Baldelli P (2010) Cortico-hippocampal hyperexcitability in synapsin I/II/III knockout mice: age-dependency and response to the antiepileptic drug levetiracetam. Neuroscience 171:268-283. CrossRef Medline

Branco T, Marra V, Staras K (2010) Examining size-strength relationships at hippocampal synapses using an ultrastructural measurement of synaptic release probability. J Struct Biol 172:203-210. CrossRef Medline

Brautigam CA, Chelliah Y, Deisenhofer J (2004) Tetramerization and ATP binding by a protein comprising the $\mathrm{A}-\mathrm{C}$ domains of rat synapsin I. J Biol Chem 279:11948-11956. CrossRef Medline

Bykhovskaia M (2011) Synapsin regulation of vesicle organization and functional pools. Semin Cell Dev Biol 22:387-392. CrossRef Medline

Cesca F, Baldelli P, Valtorta F, Benfenati F (2010) The synapsins: key actors of synapse function and plasticity. Prog Neurobiol 91:313-348. CrossRef Medline

Chi P, Greengard P, Ryan TA (2001) Synapsin dispersion and reclustering during synaptic activity. Nat Neurosci 4:1187-1193. CrossRef Medline

Chi P, Greengard P, Ryan TA (2003) Synaptic vesicle mobilization is regulated by distinct synapsin I phosphorylation pathways at different frequencies. Neuron 38:69-78. CrossRef Medline

Chouhan AK, Ivannikov MV, Lu Z, Sugimori M, Llinás RR, Macleod GT (2012) Cytosolic calcium coordinates mitochondrial energy metabolism with presynaptic activity. J Neurosci 32:1233-1243. CrossRef Medline

Coleman WL, Bill CA, Simsek-Duran F, Lonart G, Samigullin D, Bykhovskaia M (2008) Synapsin II and calcium regulate vesicle docking and the cross-talk between vesicle pools at the mouse motor terminals. J Physiol 586:4649-4673. CrossRef Medline

Darcy KJ, Staras K, Collinson LM, Goda Y (2006) Constitutive sharing of recycling synaptic vesicles between presynaptic boutons. Nat Neurosci 9:315-321. CrossRef Medline

Denker A, Rizzoli SO (2010) Synaptic vesicle pools: an update. Front Synaptic Neurosci 2:135. CrossRef Medline

Esser L, Wang CR, Hosaka M, Smagula CS, Südhof TC, Deisenhofer J (1998) Synapsin I is structurally similar to ATP-utilizing enzymes. EMBO J 17: 977-984. CrossRef Medline

Gabriel T, García-Pérez E, Mahfooz K, Goñi J, Martínez-Turrillas R, PérezOtaño I, Lo DC, Wesseling JF (2011) A new kinetic framework for synaptic vesicle trafficking tested in synapsin knock-outs. J Neurosci 31: 11563-11577. CrossRef Medline

García-Pérez E, Lo DC, Wesseling JF (2008) Kinetic isolation of a slowly recovering component of short-term depression during exhaustive use at excitatory hippocampal synapses. J Neurophysiol 100:781-795. CrossRef Medline

Gitler D, Xu Y, Kao HT, Lin D, Lim S, Feng J, Greengard P, Augustine GJ (2004a) Molecular determinants of synapsin targeting to presynaptic terminals. J Neurosci 24:3711-3720. CrossRef Medline

Gitler D, Takagishi Y, Feng J, Ren Y, Rodriguiz RM, Wetsel WC, Greengard P, Augustine GJ (2004b) Different presynaptic roles of synapsins at excitatory and inhibitory synapses. J Neurosci 24:11368-11380. CrossRef Medline

Gitler D, Cheng Q, Greengard P, Augustine GJ (2008) Synapsin IIa controls the reserve pool of glutamatergic synaptic vesicles. J Neurosci 28:1083510843. CrossRef Medline

Glancy B, Balaban RS (2012) Role of mitochondrial Ca2+ in the regulation of cellular energetics. Biochemistry 51:2959-2973. CrossRef Medline

Gordon SL, Cousin MA (2014) The Sybtraps: control of synaptobrevin traffic by synaptophysin, alpha-synuclein and AP-180. Traffic 15:245-254. CrossRef Medline

Gribble FM, Loussouarn G, Tucker SJ, Zhao C, Nichols CG, Ashcroft FM (2000) A novel method for measurement of submembrane ATP concentration. J Biol Chem 275:30046-30049. CrossRef Medline 
Groh A, de Kock CP, Wimmer VC, Sakmann B, Kuner T (2008) Driver or coincidence detector: modal switch of a corticothalamic giant synapse controlled by spontaneous activity and short-term depression. J Neurosci 28:9652-9663. CrossRef Medline

Hackett JT, Cochran SL, Greenfield LJ Jr, Brosius DC, Ueda T (1990) Synapsin I injected presynaptically into goldfish mauthner axons reduces quantal synaptic transmission. J Neurophysiol 63:701-706. Medline

Harris JJ, Jolivet R, Attwell D (2012) Synaptic energy use and supply. Neuron 75:762-777. CrossRef Medline

Henquin JC (2000) Triggering and amplifying pathways of regulation of insulin secretion by glucose. Diabetes 49:1751-1760. CrossRef Medline

Hilfiker S, Schweizer FE, Kao HT, Czernik AJ, Greengard P, Augustine GJ (1998) Two sites of action for synapsin domain E in regulating neurotransmitter release. Nat Neurosci 1:29-35. CrossRef Medline

Hilfiker S, Benfenati F, Doussau F, Nairn AC, Czernik AJ, Augustine GJ, Greengard P (2005) Structural domains involved in the regulation of transmitter release by synapsins. J Neurosci 25:2658-2669. CrossRef Medline

Hosaka M, Südhof TC (1998a) Synapsin III, a novel synapsin with an unusual regulation by $\mathrm{Ca}^{2+}$. J Biol Chem 273:13371-13374. CrossRef Medline

Hosaka M, Südhof TC (1998b) Synapsins I and II are ATP-binding proteins with differential $\mathrm{Ca}^{2+}$ regulation. J Biol Chem 273:1425-1429. CrossRef Medline

Hosaka M, Südhof TC (1999) Homo- and heterodimerization of synapsins. J Biol Chem 274:16747-16753. CrossRef Medline

Hosaka M, Hammer RE, Südhof TC (1999) A phospho-switch controls the dynamic association of synapsins with synaptic vesicles. Neuron 24:377387. CrossRef Medline

Imamura H, Nhat KP, Togawa H, Saito K, Iino R, Kato-Yamada Y, Nagai T, Noji H (2009) Visualization of ATP levels inside single living cells with fluorescence resonance energy transfer-based genetically encoded indicators. Proc Natl Acad Sci U S A 106:15651-15656. CrossRef Medline

Johnston PA, Jahn R, Südhof TC (1989) Transmembrane topography and evolutionary conservation of synaptophysin. J Biol Chem 264:12681273. Medline

Jovanovic JN, Sihra TS, Nairn AC, Hemmings HC Jr, Greengard P, Czernik AJ (2001) Opposing changes in phosphorylation of specific sites in synapsin I during $\mathrm{Ca}^{2+}$-dependent glutamate release in isolated nerve terminals. J Neurosci 21:7944-7953. Medline

Kaeser PS, Regehr WG (2014) Molecular mechanisms for synchronous, asynchronous, and spontaneous neurotransmitter release. Annu Rev Physiol 76:333-363. CrossRef Medline

Kamin D, Lauterbach MA, Westphal V, Keller J, Schönle A, Hell SW, Rizzoli SO (2010) High- and low-mobility stages in the synaptic vesicle cycle. Biophys J 99:675-684. CrossRef Medline

Kao HT, Porton B, Hilfiker S, Stefani G, Pieribone VA, DeSalle R, Greengard P (1999) Molecular evolution of the synapsin gene family. J Exp Zool 285:360-377. CrossRef Medline

Kim SH, Ryan TA (2013) Balance of calcineurin Aalpha and CDK5 activities sets release probability at nerve terminals. J Neurosci 33:8937-8950. CrossRef Medline

Kwon SE, Chapman ER (2011) Synaptophysin regulates the kinetics of synaptic vesicle endocytosis in central neurons. Neuron 70:847-854. CrossRef Medline

Laviv T, Riven I, Dolev I, Vertkin I, Balana B, Slesinger PA, Slutsky I (2010) Basal GABA regulates GABA(B)R conformation and release probability at single hippocampal synapses. Neuron 67:253-267. CrossRef Medline

Li Z, Murthy VN (2001) Visualizing postendocytic traffic of synaptic vesicles at hippocampal synapses. Neuron 31:593-605. CrossRef Medline

Llinás R, McGuinness TL, Leonard CS, Sugimori M, Greengard P (1985) Intraterminal injection of synapsin I or calcium/calmodulin-dependent protein kinase II alters neurotransmitter release at the squid giant synapse. Proc Natl Acad Sci U S A 82:3035-3039. CrossRef Medline

MacAskill AF, Atkin TA, Kittler JT (2010) Mitochondrial trafficking and the provision of energy and calcium buffering at excitatory synapses. Eur J Neurosci 32:231-240. CrossRef Medline

Marchetti M, Bruno S, Campanini B, Peracchi A, Mai N, Mozzarelli A (2013) ATP binding to human serine racemase is cooperative and modulated by glycine. Febs J 280:5853-5863. CrossRef Medline

Medrihan L, Cesca F, Raimondi A, Lignani G, Baldelli P, Benfenati F (2013)
Synapsin II desynchronizes neurotransmitter release at inhibitory synapses by interacting with presynaptic calcium channels. Nat Commun 4:1512. CrossRef Medline

Menegon A, Bonanomi D, Albertinazzi C, Lotti F, Ferrari G, Kao HT, Benfenati F, Baldelli P, Valtorta F (2006) Protein kinase A-mediated synapsin I phosphorylation is a central modulator of $\mathrm{Ca}^{2+}$-dependent synaptic activity. J Neurosci 26:11670-11681. CrossRef Medline

Mozhayeva MG, Matos MF, Liu X, Kavalali ET (2004) Minimum essential factors required for vesicle mobilization at hippocampal synapses. J Neurosci 24:1680-1688. CrossRef Medline

Nagai T, Ibata K, Park ES, Kubota M, Mikoshiba K, Miyawaki A (2002) A variant of yellow fluorescent protein with fast and efficient maturation for cell-biological applications. Nat Biotechnol 20:87-90. CrossRef Medline

Neher E (2010) What is rate-limiting during sustained synaptic activity: vesicle supply or the availability of release sites. Frontiers in Synaptic Neuroscience 2 .

Neher E (2012) Introduction: regulated exocytosis. Cell Calcium 52:196198. CrossRef Medline

Orenbuch A, Shalev L, Marra V, Sinai I, Lavy Y, Kahn J, Burden JJ, Staras K, Gitler D (2012a) Synapsin selectively controls the mobility of resting pool vesicles at hippocampal terminals. J Neurosci 32:3969-3980. CrossRef Medline

Orenbuch A, Ayelet O, Shulman Y, Yoav S, Lipstein N, Noa L, Bechar A, Amit B, Lavy Y, Yotam L, Brumer E, Eliaz B, Vasileva M, Mariya V, Kahn J, Joy K, Barki-Harrington L, Liza BH, Kuner T, Thomas K, Gitler D, Daniel G (2012b) Inhibition of exocytosis or endocytosis blocks activity-dependent redistribution of synapsin. J Neurochem 120:248-258. CrossRef Medline

Prendergast FG, Mann KG (1978) Chemical and physical properties of aequorin and the green fluorescent protein isolated from Aequorea forskalea. Biochemistry 17:3448-3453. CrossRef Medline

Rangaraju V, Calloway N, Ryan TA (2014) Activity-driven local ATP synthesis is required for synaptic function. Cell 156:825-835. CrossRef Medline

Regehr WG (2012) Short-term presynaptic plasticity. Cold Spring Harb Perspect Biol 4:a005702. CrossRef Medline

Rizzoli SO (2014) Synaptic vesicle recycling: steps and principles. EMBO J 33:788-822. CrossRef Medline

Rosahl TW, Spillane D, Missler M, Herz J, Selig DK, Wolff JR, Hammer RE, Malenka RC, Südhof TC (1995) Essential functions of synapsins I and II in synaptic vesicle regulation. Nature 375:488-493. CrossRef Medline

Saka S, Rizzoli SO (2012) Super-resolution imaging prompts re-thinking of cell biology mechanisms: selected cases using stimulated emission depletion microscopy. Bioessays 34:386-395. CrossRef Medline

Samigullin D, Bill CA, Coleman WL, Bykhovskaia M (2004) Regulation of transmitter release by synapsin II in mouse motor terminals. J Physiol 561:149-158. CrossRef Medline

Schoenmakers TJ, Visser GJ, Flik G, Theuvenet AP (1992) CHELATOR: an improved method for computing metal ion concentrations in physiological solutions. Biotechniques 12:870-874, 876-879. Medline

Staras K, Branco T, Burden JJ, Pozo K, Darcy K, Marra V, Ratnayaka A, Goda Y (2010) A vesicle superpool spans multiple presynaptic terminals in hippocampal neurons. Neuron 66:37-44. CrossRef Medline

Staras K, Mikulincer D, Gitler D (2013) Monitoring and quantifying dynamic physiological processes in live neurons using fluorescence recovery after photobleaching. J Neurochem 126:213-222. CrossRef Medline

Stölting G, Teodorescu G, Begemann B, Schubert J, Nabbout R, Toliat MR, Sander T, Nürnberg P, Lerche H, Fahlke C (2013) Regulation of ClC-2 gating by intracellular ATP. Pflugers Arch 465:1423-1437. CrossRef Medline

Südhof TC (2004) The synaptic vesicle cycle. Annu Rev Neurosci 27:509_ 547. CrossRef Medline

Svichar N, Esquenazi S, Chen HY, Chesler M (2011) Preemptive regulation of intracellular $\mathrm{pH}$ in hippocampal neurons by a dual mechanism of depolarization-induced alkalinization. J Neurosci 31:6997-7004. CrossRef Medline

Thiel G, Südhof TC, Greengard P (1990) Synapsin II. Mapping of a domain in the NH2-terminal region which binds to small synaptic vesicles. J Biol Chem 265:16527-16533. Medline

Toloe J, Mollajew R, Kügler S, Mironov SL (2014) Metabolic differences in 
hippocampal 'Rett' neurons revealed by ATP imaging. Mol Cell Neurosci 59:47-56. CrossRef Medline

Verstegen AM, Tagliatti E, Lignani G, Marte A, Stolero T, Atias M, Corradi A, Valtorta F, Gitler D, Onofri F, Fassio A, Benfenati F (2014) Phosphorylation of synapsin I by cyclin-dependent kinase- 5 sets the ratio between the resting and recycling pools of synaptic vesicles at hippocampal synapses. J Neurosci 34:7266-7280. CrossRef Medline

Walter AM, Pinheiro PS, Verhage M, Sørensen JB (2013) A sequential vesicle pool model with a single release sensor and a $\mathrm{Ca}(2+)$-dependent priming catalyst effectively explains $\mathrm{Ca}(2+)$-dependent properties of neurosecretion. PLoS Comput Biol 9:e1003362. CrossRef Medline

Wilson JE, Chin A (1991) Chelation of divalent cations by ATP, studied by titration calorimetry. Anal Biochem 193:16-19. CrossRef Medline

Yaniv Y, Juhaszova M, Nuss HB, Wang S, Zorov DB, Lakatta EG, Sollott SJ (2010) Matching ATP supply and demand in mammalian heart: in vivo, in vitro, and in silico perspectives. Ann N Y Acad Sci 1188:133-142. CrossRef Medline

Zhang Z, Nguyen KT, Barrett EF, David G (2010) Vesicular ATPase inserted into the plasma membrane of motor terminals by exocytosis alkalinizes cytosolic pH and facilitates endocytosis. Neuron 68:1097-1108. CrossRef Medline

Zucker RS, Regehr WG (2002) Short-term synaptic plasticity. Annual Review of Physiology 64:355-405. CrossRef Medline 